Research Article

\title{
Charmless $B_{c} \rightarrow P P, P V$ Decays in the QCD Factorization Approach
}

\author{
Na Wang \\ Institute of Particle Physics and Key Laboratory of Quark and Lepton Physics (MOE), \\ Central China Normal University, Wuhan, Hubei 430079, China \\ Correspondence should be addressed to Na Wang; wangna05001@126.com
}

Received 22 February 2016; Accepted 9 May 2016

Academic Editor: Juan José Sanz-Cillero

Copyright (C) $2016 \mathrm{Na}$ Wang. This is an open access article distributed under the Creative Commons Attribution License, which permits unrestricted use, distribution, and reproduction in any medium, provided the original work is properly cited. The publication of this article was funded by SCOAP S $^{3}$

\begin{abstract}
The charmless $B_{c} \rightarrow P P, P V$ decays (where $P$ and $V$ denote the light pseudoscalar and vector mesons, resp.) can occur only via the weak annihilation diagrams within the Standard Model. In this paper, we study these kinds of decays in the framework of QCD factorization, by adopting two different schemes: scheme I is similar to the method usually adopted in the QCD factorization approach, while scheme II is based on the infrared behavior of gluon propagator and running coupling. For comparison, in our calculation, we adopt three kinds of wave functions for $B_{c}$ meson. The branching ratios based on the two schemes are given. It is found that (a) the predicted branching ratios in scheme I are, however, quite small and almost impossible to be measured at the LHCb experiment and (b) in scheme II, by assigning a dynamical gluon mass to the gluon propagator, we can avoid enhancements of the contribution from soft endpoint region. The strength of annihilation contributions predicted in scheme II is enhanced compared to that obtained in scheme I.
\end{abstract}

\section{Introduction}

The $B_{c}$ meson is the lowest-lying bound state of two heavy quarks with different flavors $(\bar{b}$ and $c)$. Due to its flavor quantum numbers $B=C= \pm 1$ and being below the $B D$ threshold, the $B_{c}$ meson is stable against strong and electromagnetic interactions and can decay only via weak interaction. Furthermore, the $B_{c}$ meson has a sufficiently large mass; each of the two heavy quarks can decay individually, resulting in rich decay channels [1]. Therefore, the $B_{c}$ meson is an ideal system to study weak decays of heavy mesons [2].

The experimental studies of $B_{c}$ meson properties started in 1998 when the Collider Detector at Fermilab (CDF) reported the first observation of $B_{c}$ meson through the semileptonic decay modes $B_{c} \rightarrow J / \Psi \ell^{+} X(\ell=e, \mu)$ [3]. Thanks to the fruitful performance of the CDF, D0, and LHCb collaborations, both the mass [4-6] and the lifetime [7-9] of the $B_{c}$ meson have been measured quite accurately. At the Large Hadron Collider (LHC) with a luminosity of about
$\mathscr{L}=10^{34} \mathrm{~cm}^{-2} \mathrm{~s}^{-1}$, one could expect around $5 \times 10^{10} B_{c}$ events per year [10]. In addition, several hadronic $B_{c}$ decay channels, such as $B_{c}^{+} \rightarrow J / \psi K^{+}[11]$ and $B_{c}^{+} \rightarrow B_{s}^{0} \pi^{+}$[12], have also been observed for the first time. In the following years, the properties of $B_{c}$ meson and the dynamics involved in $B_{c}$ decays will be further exploited through the precision measurements at the LHC with its high collision energy and high luminosity, opening therefore a golden era of $B_{c}$ physics [13].

The theoretical investigations have also been carried out on the properties of $B_{c}$ meson, such as its lifetime, its decay constant, and some of its form factors, based on different theoretical frameworks [2]. Due to its heavy-heavy nature and the participation of strong interaction, the hadronic $B_{c}$ decays are extremely complicated but, at the same time, provide great opportunities to study the perturbative and nonperturbative QCD and final-state interactions in heavy meson decays. Being weakly decaying and doubly heavy flavor meson, it also offers a novel window for studying the heavy-quark dynamics that is inaccessible through the $b \bar{b}$ and $c \bar{c}$ quarkonia [2]. These features have motivated an extensive 
study of $B_{c}$ decays in various theoretical approaches in the literature [14].

In this paper, we will focus on the two-body charmless hadronic $B_{c}$ decays, which can proceed only via the weak annihilation diagrams in the Standard Model (SM): the initial $\bar{b}$ and $c$ quarks annihilate into $u$ and $\bar{d} / \bar{s}$ quarks, which form two light mesons by hadronizing with $q \bar{q}(q=u, d, s)$ pair emitted from a gluon. Detailed studies of these decays will be certainly helpful for further improving our understanding of the weak annihilation contributions, the size of which is currently an important issue in $B$ physics.

The recent measurements of the $B_{u, d, s}$ decays, especially of the pure annihilation processes $B_{s} \rightarrow \pi^{+} \pi^{-}$and $B_{d} \rightarrow$ $K^{+} K^{-}$[15-17], indicate that the annihilation topologies can be significant, contrary to the common belief of their power suppression in the heavy-quark limit [18]. Although it was later noticed theoretically that the annihilation amplitudes may not be negligibly small in realistic $B$ meson decays [19], it is still very hard to make a reliable calculation of these diagrams, and quantitative predictions for them vary greatly between different approaches. In the QCD factorization (QCDF) approach [20], they can only be estimated in a model-dependent way due to the endpoint singularities [21]. In the soft-collinear effective theory (SCET) [22], they are argued to be factorizable and almost real with tiny strong phase [23], which is rather different from almost imaginary with large strong phase as predicted in the perturbative QCD approach (pQCD) [19]. In addition, the annihilation contributions in many $B_{u, d, s}$ decays usually involve both tree and penguin operators, and they interfere with many other different topologies, making it difficult to obtain an accurate value of annihilation by fitting the experimental data [24].

The charmless $B_{c}$ decays into two light mesons, coming only from a single tree operator, provide therefore an ideal testing ground for annihilation in heavy meson decays and deserve detailed studies using different theoretical approaches [25-28]. In this paper, we will revisit these decays in the QCDF framework, using two different schemes proposed to deal with the endpoint singularity and to avoid enhancements in the soft endpoint region: the divergence in scheme I is usually parameterized with at least two phenomenological parameters through the treatment $\int_{0}^{1} d x / x \rightarrow$ $X_{A, H}=\ln \left(m_{B} / \Lambda_{h}\right)\left(1+\rho_{A, H} e^{i \phi_{A, H}}\right)$ [21], whereas in scheme II, one could use an infrared finite gluon propagator $1 /\left(k^{2}+\right.$

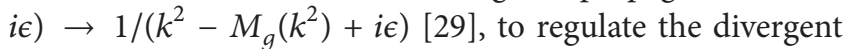
integrals [30-34]. The different scenarios corresponding to different choices of $\rho_{A, H}$ and $\phi_{A, H}$ in scheme I have been thoroughly discussed in [21]. In scheme II, it is found that the hard spectator-scattering contributions are real and the annihilation corrections are complex with a large imaginary part [30, 31, 34]. These two different treatments used in $B_{u, d, s}$ decays could be further tested through the charmless $B_{c}$ decays.

The remaining parts of the paper are organized as follows. In Section 2, after recapitulating the theoretical framework for two-body charmless hadronic $B_{c}$ decays, we present the calculation of the annihilation diagrams in the QCDF framework with the two different schemes. The numerical results and discussions are given in Section 3. Finally, Section 4 contains the main conclusions and a short summary. The explicit expressions for the decay amplitudes and the relevant input parameters are collected in Appendices A and $\mathrm{B}$, respectively.

\section{Theoretical Framework and Calculation}

2.1. The Effective Weak Hamiltonian and Hadronic Matrix Element. Using the operator product expansion and renormalization group (RG) equation, we can write the effective weak Hamiltonian for charmless $B_{c}^{-} \rightarrow M_{1} M_{2}\left(M_{i}\right.$ denote the light pseudoscalar and vector mesons) decays as [35]

$$
\mathscr{H}_{\text {eff }}=\frac{G_{F}}{\sqrt{2}} V_{c b} V_{u D}^{*}\left[C_{1}(\mu) Q_{1}+C_{2}(\mu) Q_{2}\right]+\text { h.c. },
$$

where $G_{F}$ is the Fermi coupling constant and $V_{c b}$ and $V_{u D}$ $(D=d, s)$ are the Cabibbo-Kobayashi-Maskawa (CKM) matrix elements [36]. The four-quark operators $Q_{i}$ arise from $\mathrm{W}$-boson exchange and are defined, respectively, as

$$
\begin{aligned}
Q_{1} & =\left[\bar{c}_{\alpha} \gamma^{\mu}\left(1-\gamma_{5}\right) b_{\alpha}\right]\left[\bar{D}_{\beta} \gamma_{\mu}\left(1-\gamma_{5}\right) u_{\beta}\right], \\
Q_{2} & =\left[\bar{c}_{\alpha} \gamma^{\mu}\left(1-\gamma_{5}\right) b_{\beta}\right]\left[\bar{D}_{\beta} \gamma_{\mu}\left(1-\gamma_{5}\right) u_{\alpha}\right],
\end{aligned}
$$

where $\alpha, \beta$ are the color indices. The corresponding Wilson coefficients $C_{i}(\mu)$ can be calculated using the RG improved perturbative theory [35].

To obtain the decay amplitude, the remaining work is to evaluate the hadronic matrix elements of the local operators $Q_{i}$, which is however quite difficult due to the participation of nonperturbative QCD effects. The Feynman diagrams for $B_{c} \rightarrow M_{1} M_{2}$ decays with the QCDF approach are shown in Figure 1, where (a), (b) and (c), (d) are nonfactorizable and factorizable topologies, respectively. Since the tree operators $Q_{1,2}$ have the $(V-A) \otimes(V-A)$ Dirac structure, the two factorizable diagrams (c) and (d) cancel each other exactly in the QCDF approach due to the conservation of the vector current and partial conservation of axial-vector current, plus the approximation that final states have the same asymptotic expressions for twist- 2 and twist- 3 distribution amplitudes (see (12)) in our calculation [21]. Moreover, due to the mismatch of the color indices, there are no contributions from diagrams (a) and (b) with the insertion of the colorsinglet operator $Q_{1}$. Thus, there is only a single tree operator $Q_{2}$ involved in the decay amplitudes, and the nonzero contribution comes only from diagrams (a) and (b).

In the QCDF framework and with the same hypotheses made for hadronic $B_{u, d, s}$ decays, the decay amplitude for charmless $B_{c}^{-} \rightarrow M_{1} M_{2}$ decays can be written as [21]

$$
\left\langle M_{1} M_{2}\left|\mathscr{H}_{\text {eff }}\right| B_{c}^{-}\right\rangle \propto f_{B_{c}} f_{M_{1}} f_{M_{2}} b_{2}\left(M_{1}, M_{2}\right),
$$

where $f_{B_{c}}, f_{M}$ are decay constants of the $B_{c}$ and $M$ mesons, respectively. The coefficient $b_{2}\left(M_{1}, M_{2}\right)$ is defined as [21]

$$
b_{2}\left(M_{1}, M_{2}\right)=\frac{C_{F}}{N_{c}^{2}} C_{2} A_{1}^{i}\left(M_{1}, M_{2}\right),
$$




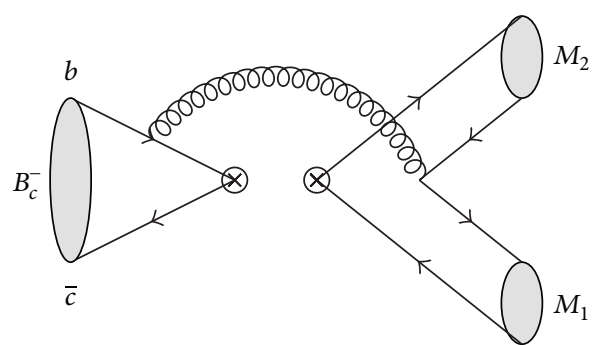

(a)
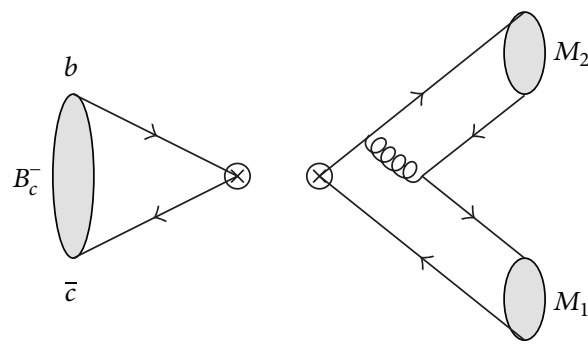

(c)

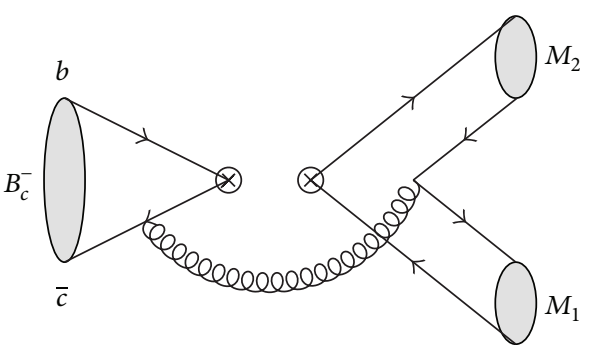

(b)
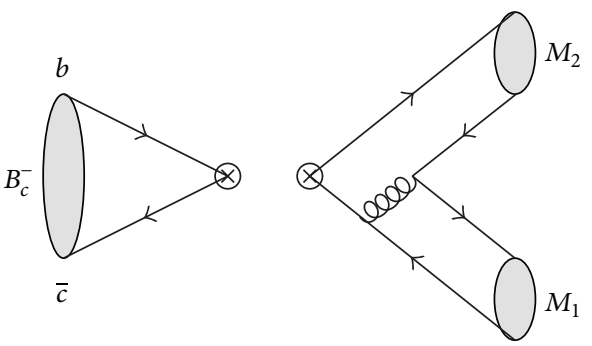

(d)

Figure 1: The lowest order Feynman diagrams contributing to charmless $B_{c}^{-} \rightarrow M_{1} M_{2}$ decays.

where $C_{F}=4 / 3$ and $N_{c}=3$, the superscript " $i$ " on $A_{1}^{i}$ refers to the gluon emission from the initial-state quarks, and the subscript "1" on $A_{1}^{i}$ refers to the $(V-A) \otimes(V-A)$ Dirac structure of the inserted four-quark operator $Q_{2}$. The basic building block $A_{1}^{i}\left(M_{1}, M_{2}\right)$ can be expressed as the convolution of the hard kernels given by diagrams (a) and (b) in Figure 1 and the light-cone distribution amplitudes (LCDAs) of the initial- and final-state mesons, which will be detailed in the next two subsections.

2.2. $A_{1}^{i}\left(M_{1}, M_{2}\right)$ in Scheme I. In scheme I, the annihilation contributions to hadronic $B_{u, d, s}$ decays are evaluated by regularizing the divergent integrals on the basis of heavyquark power counting [21]. Despite the fact that such a treatment is not entirely self-consistent in the context of a hard-scattering approach, it provides nevertheless a model to estimate the importance of annihilation, which, motivated by the first observation of the pure annihilation decay $B_{s} \rightarrow$ $\pi^{+} \pi^{-}$[15-17], has been revisited quite recently in [31, 37].

Following a similar treatment, we now estimate the annihilation topologies in charmless $B_{c}^{-} \rightarrow M_{1} M_{2}$ decays. In accordance with the convention adopted in [21], we find that the basic building block $A_{1}^{i}\left(M_{1}, M_{2}\right)$ is given by

$$
\begin{gathered}
A_{1}^{i}\left(M_{1}, M_{2}\right)=\pi \alpha_{s} \int_{0}^{1} d x d y d z \Phi_{M_{B_{c}}}(z)\left\{\Phi_{M_{2}}(x)\right. \\
\cdot \Phi_{M_{1}}(y)\left[\frac{\bar{x}-\bar{z}+z_{b}}{\bar{x} y[(\bar{x}+y) \bar{z}-\bar{x} y-i \epsilon]}\right. \\
\left.-\frac{y-z+z_{c}}{\bar{x} y[(\bar{x}+y) z-\bar{x} y-i \epsilon]}\right]+r_{\chi}^{M_{1}} r_{\chi}^{M_{2}} \Phi_{m_{2}}(x)
\end{gathered}
$$

$$
\begin{aligned}
& \cdot \Phi_{m_{1}}(y)\left[\frac{\bar{x} y z-x \bar{y} \bar{z}+z_{b}}{\bar{x} y[(\bar{x}+y) \bar{z}-\bar{x} y-i \epsilon]}\right. \\
& \left.\left.-\frac{\bar{x} y \bar{z}-x \bar{y} z+z_{c}}{\bar{x} y[(\bar{x}+y) z-\bar{x} y-i \epsilon]}\right]\right\},
\end{aligned}
$$

when both mesons are pseudoscalar or when $M_{1}$ is a pseudoscalar and $M_{2}$ a vector meson. In the case when $M_{1}$ is a vector meson and $M_{2}$ a pseudoscalar, one has to change the sign of the second term in $A_{1}^{i}$. When we take $\bar{z}=z_{b}=1$ and $z=z_{c}=0$, this result is in agreement with the expressions obtained in [21,38]. In (5), $z_{b}$ and $z_{c}$ denote the relative size of the $b$ and $c$ quark masses with

$$
\begin{aligned}
& z_{b}=\frac{m_{b}}{m_{B_{c}}}, \\
& z_{c}=\frac{m_{c}}{m_{B_{c}}} .
\end{aligned}
$$

Their appearance allows one to distinguish the origin of each term in the brackets: the ones involving $z_{b}$ must come from diagram (a), whereas those involving $z_{c}$ must come from diagram (b) in Figure 1. As always, $\Phi_{M}(x)$ and $\Phi_{m}(x)$ denote the leading-twist and twist-3 two-particle LCDAs of the final-state meson $M$, respectively. The factor $r_{\chi}^{M}$, once multiplied by $m_{B_{c}} / 2$, is used to normalize the twist-3 distribution amplitude; explicitly, we have

$$
\begin{aligned}
& r_{\chi}^{P}(\mu)=\frac{2 m_{P}^{2}}{m_{B_{c}}\left[m_{1}(\mu)+m_{2}(\mu)\right]}, \\
& r_{\chi}^{V}(\mu)=\frac{2 m_{V}}{m_{B_{c}}} \frac{f_{V}^{\perp}(\mu)}{f_{V}},
\end{aligned}
$$




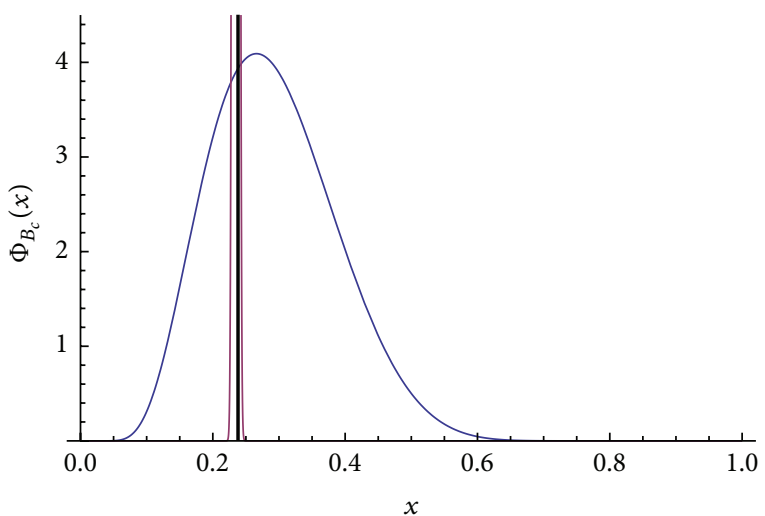

FIGURE 2: The three kinds of distribution function for $B_{c}$ meson, W-I thick solid line, W-II blue line, and W-III red line.

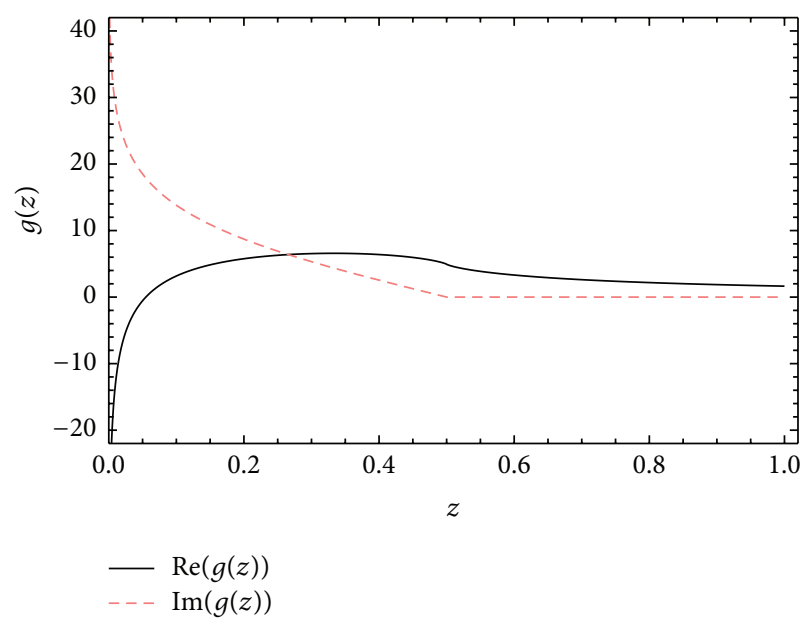

FIGURE 3: The variation of the real and imaginary parts of function $g(z)$ defined by (13) with respect to the parameter $z$.

To pursue the structure of the singularities of the building block $A_{1}^{i}\left(M_{1}, M_{2}\right)$, we take, for simplicity, the asymptotic expressions for the distribution amplitudes [21, 43]

$$
\begin{aligned}
& \Phi_{P}(x)=6 x(1-x), \\
& \Phi_{V}(x)=6 x(1-x), \\
& \Phi_{p}(x)=1, \\
& \Phi_{v}(x)=3(2 x-1) .
\end{aligned}
$$

The weak annihilation of $B_{u, d, s}$ exhibits endpoint singularities even at twist-2 order in the light-cone expansion for the final-state mesons. For $B_{c} \rightarrow P P, P V$ decays, the endpoint singularities only at twist- 3 level, the situation is the same as the hard spectator interactions of $B_{u, d, s}$. For the twist-2 terms, the singularities are in the integration interval. It is found that the convolution integrals in (5) can be performed without problem as long as $1 / 2 \leq z<1$. There are, however, integrable singularities at $\bar{x}=z /(1-z)$ or $y=z /(1-z)$ when $0<z<1 / 2$, which can be dealt with using the prescription of Cauchy principal value integral. Taking the integral

$$
g(z)=\int_{0}^{1} d x d y \frac{1}{(\bar{x}+y) z-\bar{x} y-i \epsilon}
$$

where $x_{B}=1-m_{b} / m_{B}$.

In (9) and (10), $N$ is normalization constant and the normalization condition is

$$
\int_{0}^{1} d x \Phi_{B_{c}}(x)=1
$$

The shape of the three distribution functions for $B_{c}$ meson are displayed in Figure 2. as an example, we show in Figure 3 its real and imaginary parts dependence on the parameter $z$, and one can see clearly 1. In (5), the twist-3 terms are more complex and can not be expressed as polynomial of $X_{A}=\int_{0}^{1} d y / y \sim \ln \left(m_{b} / \Lambda_{\mathrm{QCD}}\right)$, so we make the integral interval of $\bar{x}, y \in\left[\Lambda_{\mathrm{QCD}} / m_{b}, 1\right]$.

Rather than giving the explicit expressions for the convolution integrals, we present, with the default inputs that the integral is finite as long as $z$ is different from 0 and 
$m_{b}=4.8 \mathrm{GeV}$ and $m_{c}=1.5 \mathrm{GeV}$, the numerical results for the building block $A_{1}^{i}\left(M_{1}, M_{2}\right)$ in the three different cases

$W-I$

$$
\begin{aligned}
& A_{1}^{i}(P, P) \\
& =\pi\left[(-5.70+6.26 i)+r_{\chi}^{M_{1}} r_{\chi}^{M_{2}}(-1.59-2.75 i)\right], \\
& A_{1}^{i}(P, V) \\
& =\pi\left[(-5.70+6.26 i)+r_{\chi}^{M_{1}} r_{\chi}^{M_{2}}(-3.73+0.56 i)\right], \\
& A_{1}^{i}(V, P) \\
& =\pi\left[(-5.70+6.26 i)-r_{\chi}^{M_{1}} r_{\chi}^{M_{2}}(3.73-0.56 i)\right],
\end{aligned}
$$

W-II

$$
\begin{aligned}
& A_{1}^{i}(P, P) \\
& \quad=\pi\left[(-2.78-4.95 i)+r_{\chi}^{M_{1}} r_{\chi}^{M_{2}}(-0.09-2.47 i)\right], \\
& \begin{array}{l}
A_{1}^{i}(P, V) \\
\quad=\pi\left[(-2.78-4.95 i)+r_{\chi}^{M_{1}} r_{\chi}^{M_{2}}(-1.00-0.04 i)\right], \\
A_{1}^{i}(V, P) \\
\quad=\pi\left[(-2.78-4.95 i)-r_{\chi}^{M_{1}} r_{\chi}^{M_{2}}(1.00+0.04 i)\right],
\end{array}
\end{aligned}
$$

W-III

$$
\begin{aligned}
& A_{1}^{i}(P, P) \\
& =\pi\left[(-5.82-6.48 i)+r_{\chi}^{M_{1}} r_{\chi}^{M_{2}}(-1.71-2.85 i)\right], \\
& A_{1}^{i}(P, V) \\
& =\pi\left[(-5.82-6.48 i)+r_{\chi}^{M_{1}} r_{\chi}^{M_{2}}(-4.03+0.49 i)\right], \\
& A_{1}^{i}(P, V) \\
& =\pi\left[(-5.82-6.48 i)-r_{\chi}^{M_{1}} r_{\chi}^{M_{2}}(4.03-0.49 i)\right],
\end{aligned}
$$

where the results are obtained with $\alpha_{s}\left(\mu \simeq m_{B_{c}} / 2\right)=0.25$ and $m_{B_{c}}=6.2745 \mathrm{GeV}$. Judging from the above expressions, the branching ratios obtained with $\mathrm{W}$-I and $\mathrm{W}$-III should be very close, and the W-II's results will be smaller. It is noted that $A_{1}^{i}(P, V)$ is identical to $A_{1}^{i}(V, P)$ in our approximation and the annihilation contribution has a large imaginary part.

2.3. $A_{1}^{i}\left(M_{1}, M_{2}\right)$ in Scheme II. Instead of being parameterized with an ad hoc model-dependent cut-off, the endpoint divergences can also be regulated with an infrared (IR) finite gluon propagator that is characterized by a dynamical gluon mass, providing therefore a natural IR regulator [29]. This has been successfully applied to various hadronic $B_{u, d, s}$ decays in [30-34]. In this subsection, we will evaluate the building block $A_{1}^{i}\left(M_{1}, M_{2}\right)$ in this scheme.

Instead of the perturbative expression $1 / q^{2}$ that is IR divergent, the IR finite gluon propagator is obtained by solving an intricate set of coupled Dyson-Schwinger equations (DSE) for pure gauge QCD, under a systematic approximation and truncation [29]. It is also noted that any IR finite gluon propagator leads to a freezing of the IR coupling constant [44], meaning that the use of an IR finite gluon propagator must be accompanied by an IR finite coupling constant. The above information about the IR behavior of QCD has also been confirmed by the most recent lattice simulations $[45,46]$ (recent reviews, together with a list of references, on DSE solutions and lattice results about the infrared finite gluon propagator and running coupling constant could be found, e.g., in [47-50]). Here we adopt the gluon propagator derived by Cornwall many years ago [29]:

$$
D\left(q^{2}\right)=\frac{1}{q^{2}+M_{g}^{2}\left(q^{2}\right)},
$$

where $q^{2}$ denotes the gluon momentum squared. The corresponding running coupling constant reads [29]

$$
\alpha_{s}\left(q^{2}\right)=\frac{4 \pi}{\beta_{0} \ln \left[\left(q^{2}+4 M_{g}^{2}\left(q^{2}\right)\right) / \Lambda_{\mathrm{QCD}}^{2}\right]},
$$

where $\beta_{0}=11-(2 / 3) n_{f}$ is the first coefficient of the QCD beta function, and $n_{f}$ the number of active quark flavors at a given scale. The dynamical gluon mass $M_{g}^{2}\left(q^{2}\right)$ is given by [29]

$$
M_{g}^{2}\left(q^{2}\right)=m_{g}^{2}\left[\frac{\ln \left(\left(q^{2}+4 m_{g}^{2}\right) / \Lambda_{\mathrm{QCD}}^{2}\right)}{\ln \left(4 m_{g}^{2} / \Lambda_{\mathrm{QCD}}^{2}\right)}\right]^{-12 / 11}
$$

where $\Lambda_{\mathrm{QCD}}=225 \mathrm{MeV}$ is the QCD scale, and $m_{g}$ the effective gluon mass with a typical value $m_{g}=0.5 \pm 0.2 \mathrm{GeV}$ [29]. It is interesting to note that similar values are found by fitting the experimental data on $B_{u, d, s}$ decays: $m_{g}=$ $0.5 \pm 0.05 \mathrm{GeV}$ from $B_{u, d}$ decays [30] and $m_{g}=0.48 \pm$ $0.02 \mathrm{GeV}$ from $B_{s}$ decays [31]. In our calculation, we take $m_{g}=0.49 \pm 0.03 \mathrm{GeV}$. As shown in Figure 3, both the gluon propagator (23) and the coupling constant (24) are IR finite and different from zero at the origin of momentum squared $q^{2}=0$. 


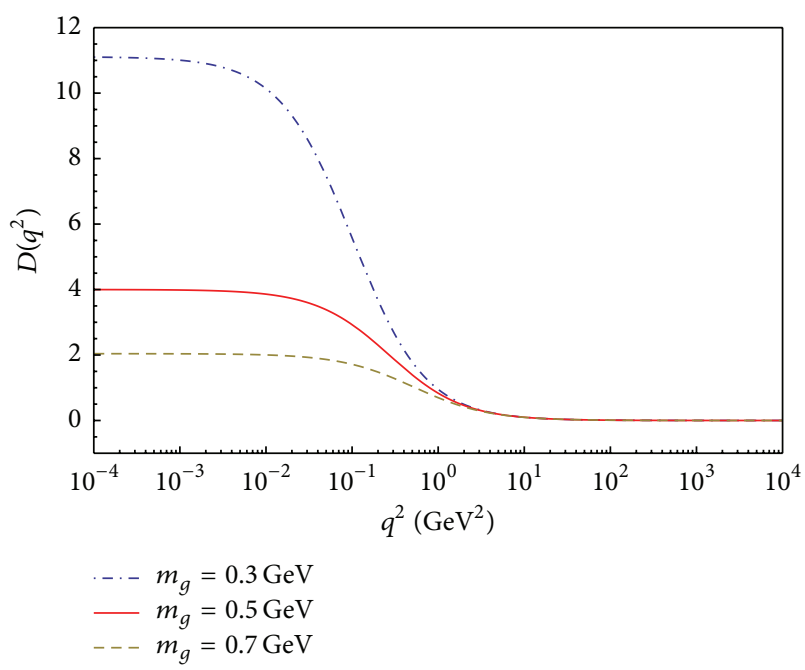

(a)

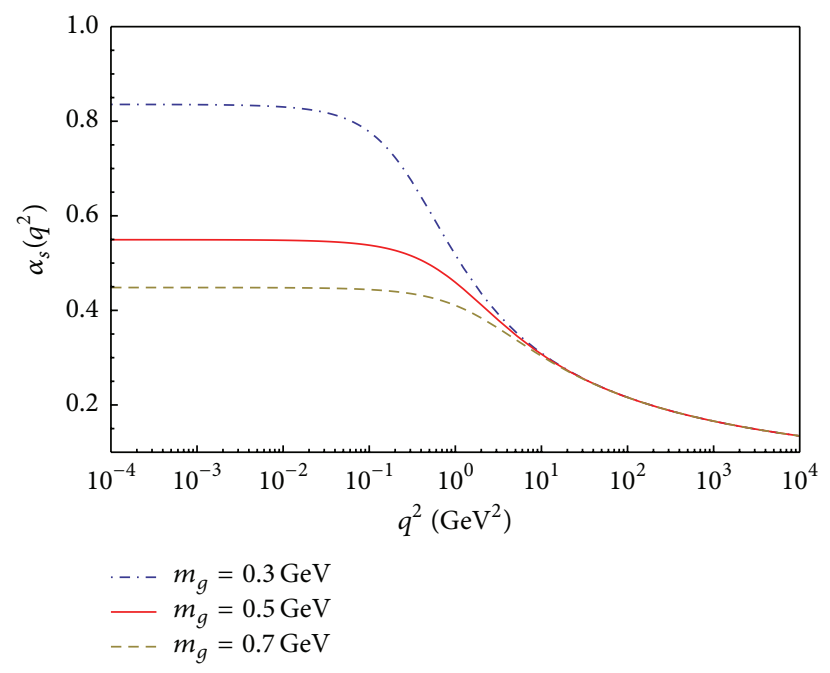

(b)

FIGURE 4: The behavior of the gluon propagator (a) and coupling constant (b) derived by Cornwall [29], with respect to the gluon momentum squared $q^{2}$.

With the above prescription and the same convention used in scheme I, our final results for the building block $A_{1}^{i}\left(M_{1}, M_{2}\right)$ can be expressed as $\left(\omega^{2}\left(q^{2}\right)=M_{g}^{2}\left(q^{2}\right) / m_{B_{c}}^{2}\right)$

$$
\begin{aligned}
& A_{1}^{i}\left(M_{1}, M_{2}\right)=\pi \int_{0}^{1} d x d y d z \alpha_{s}\left(q^{2}\right) \Phi_{M_{B_{c}}}(z) \\
& \quad \cdot\left\{\Phi_{M_{2}}(x) \Phi_{M_{1}}(y)\right. \\
& \quad \cdot\left[\frac{\bar{x}-\bar{z}+z_{b}}{\left(\bar{x} y-\omega^{2}\left(q^{2}\right)+i \epsilon\right)[(\bar{x}+y) \bar{z}-\bar{x} y-i \epsilon]}\right. \\
& \left.\quad-\frac{y-z+z_{c}}{\left(\bar{x} y-\omega^{2}\left(q^{2}\right)+i \epsilon\right)[(\bar{x}+y) z-\bar{x} y-i \epsilon]}\right] \\
& +r_{\chi}^{M_{1}} r_{x}^{M_{2}} \Phi_{m_{2}}(x) \Phi_{m_{1}}(y) \\
& \cdot\left[\frac{\bar{x} y z-x \bar{y} \bar{z}+z_{b}}{\left(\bar{x} y-\omega^{2}\left(q^{2}\right)+i \epsilon\right)[(\bar{x}+y) \bar{z}-\bar{x} y-i \epsilon]}\right. \\
& \left.\left.-\frac{\bar{x} y \bar{z}-x \bar{y} z+z_{c}}{\left(\bar{x} y-\omega^{2}\left(q^{2}\right)+i \epsilon\right)[(\bar{x}+y) z-\bar{x} y-i \epsilon]}\right]\right\},
\end{aligned}
$$

when $\left(M_{1}, M_{2}\right)=(P, P)$ and $(P, V)$. If $\left(M_{1}, M_{2}\right)=(V, P)$, on the other hand, the sign of the second term in $A_{1}^{i}$ has to be changed. When taking the limits $\bar{z}=z_{b} \rightarrow 1$ and $z=z_{c} \rightarrow 0$, our results agree with the ones given in $[30,31,34]$.

In (26), the time-like gluon momentum squared is given by $q^{2}=\bar{x} y m_{B_{c}}^{2}$ and also depends on the longitudinal momentum fractions $\bar{x}=1-x$ and $y$, making the convolution integrals rather complicated. As shown in Figure 5, although the running coupling constant is rather large in the small $q^{2}$ region (see Figure $4(\mathrm{~b})$ ), the fact that only a small fraction comes from the $q^{2}<m_{g}^{2}$ region in the $(\bar{x}, y)$ plane indicates that the annihilation contributions are still dominated by the

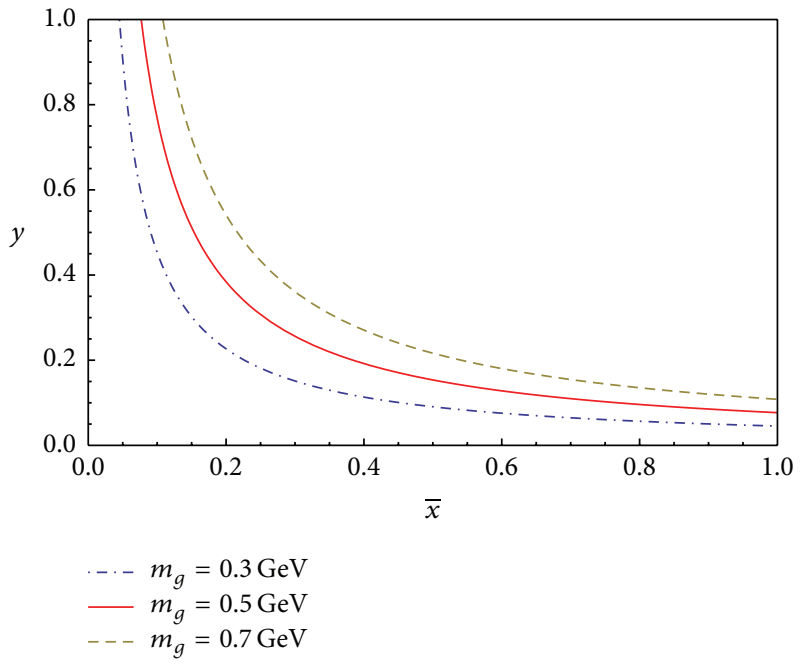

FIGURE 5: The distribution in the $(\bar{x}, y)$ plane of the IR finite gluon propagator appearing in the annihilation diagrams for charmless hadronic $B_{c} \rightarrow M_{1} M_{2}$ decays.

$q^{2}>m_{g}^{2}$ region associated with a large imaginary part [30]. In scheme II, we also use three kinds of $B_{c}$ meson wave function in the calculation. With the default inputs $m_{B_{c}}=6.2745 \mathrm{GeV}$, $m_{g}=0.49 \mathrm{GeV}, m_{b}=4.8 \mathrm{GeV}$, and $m_{c} \stackrel{B_{c}}{=} 1.5 \mathrm{GeV}$, our numerical results for the building block $A_{1}^{i}\left(M_{1}, M_{2}\right)$ read

$W-I$

$$
\begin{aligned}
& A_{1}^{i}(P, P) \\
& \quad=\pi\left[(-10.11-6.16 i)+r_{\chi}^{M_{1}} r_{\chi}^{M_{2}}(-3.64-2.36 i)\right],
\end{aligned}
$$




$$
\begin{aligned}
& A_{1}^{i}(P, V) \\
& \quad=\pi\left[(-10.11-6.16 i)+r_{\chi}^{M_{1}} r_{\chi}^{M_{2}}(-6.38+3.09 i)\right], \\
& A_{1}^{i}(V, P) \\
& \quad=\pi\left[(-10.11-6.16 i)-r_{\chi}^{M_{1}} r_{\chi}^{M_{2}}(6.38-3.09 i)\right],
\end{aligned}
$$

$W-I I$

$$
\begin{aligned}
& A_{1}^{i}(P, P) \\
& =\pi\left[(-5.84-6.06 i)+r_{\chi}^{M_{1}} r_{\chi}^{M_{2}}(-4.24-7.76 i)\right], \\
& A_{1}^{i}(P, V) \\
& =\pi\left[(-5.84-6.06 i)+r_{\chi}^{M_{1}} r_{\chi}^{M_{2}}(-18.36-9.27 i)\right], \\
& A_{1}^{i}(V, P) \\
& =\pi\left[(-5.84-6.06 i)-r_{\chi}^{M_{1}} r_{\chi}^{M_{2}}(12.5+9.94 i)\right],
\end{aligned}
$$

W-III

$$
\begin{aligned}
& A_{1}^{i}(P, P) \\
& =\pi\left[(-10.37-6.36 i)+r_{\chi}^{M_{1}} r_{\chi}^{M_{2}}(-3.57-1.89 i)\right], \\
& A_{1}^{i}(P, V) \\
& =\pi\left[(-10.37-6.36 i)+r_{\chi}^{M_{1}} r_{\chi}^{M_{2}}(-5.97+4.29 i)\right], \\
& A_{1}^{i}(V, P) \\
& =\pi\left[(-10.37-6.36 i)-r_{\chi}^{M_{1}} r_{\chi}^{M_{2}}(6.02-4.30 i)\right] .
\end{aligned}
$$

It is found that, compared to the values obtained in scheme I ((16)-(22)), the annihilation contributions predicted in scheme II are enhanced. These will apparently affect the predictions for charmless hadronic $B_{c} \rightarrow M_{1} M_{2}$ decays, which will be detailed in the next section.

\section{Numerical Results and Discussions}

In the $B_{c}$ meson rest frame, the branching ratio for general charmless $B_{c}^{-} \rightarrow M_{1} M_{2}$ decays can be written as

$$
\operatorname{Br}\left(B_{c}^{-} \longrightarrow M_{1} M_{2}\right)=\frac{\tau_{B_{c}}}{8 \pi} \frac{|\vec{p}|}{m_{B_{c}}^{2}}\left|\mathscr{A}\left(B_{c}^{-} \longrightarrow M_{1} M_{2}\right)\right|^{2},
$$

where $\tau_{B_{c}}$ is the $B_{c}$ meson lifetime and $|\vec{p}|$ is the center-ofmass momentum of either of the two outgoing mesons, with

$$
=\frac{\sqrt{\left[m_{B_{c}}^{2}-\left(m_{M_{1}}+m_{M_{2}}\right)^{2}\right]\left[m_{B_{c}}^{2}-\left(m_{M_{1}}-m_{M_{2}}\right)^{2}\right]}}{2 m_{B_{c}}} .
$$

The decay amplitude $\mathscr{A}\left(B_{c}^{-} \rightarrow M_{1} M_{2}\right)$ can be obtained from the hadronic matrix element $\left\langle M_{1} M_{2}\left|\mathscr{H}_{\text {eff }}\right| B_{c}^{-}\right\rangle$defined in (3); for convenience, we collect in Appendix A the explicit expressions of the decay amplitudes for the considered decay modes. The CP-violating asymmetries for all the considered $B_{c}$ decays are absent, since there involves only a single tree operator in the decay amplitudes, which can be clearly seen from (3).

With the theoretical expressions given above and the input parameters collected in Appendix B, we proceed to evaluate the $\mathrm{CP}$-averaged branching ratios for these charmless $B_{c} \rightarrow M_{1} M_{2}$ decays. In our calculation, the default value of the renormalization scale is set at $\mu=m_{B_{c}} / 2$, which is approximately the averaged virtuality of the time-like gluon propagated in the annihilation diagrams. The numerical results based on the two schemes are collected in Table 2. In Tables 3, 4, and 5, we also present detailed error estimates induced by the theoretical uncertainties of input parameters. The first error shown corresponds to the variation of the CKM parameters $A$ and $\lambda$ (named as "CKM"); the second error refers to the variation of the quark masses, decay constants, and the $\eta-\eta^{\prime}$ mixing angle (named as "hadronic"). The third error arises from the variation of the renormalization scale $\mu$ (named as "scale"). The last error reflects the uncertainty due to the dynamical gluon mass $m_{g}$ (named as " $m_{g}$ ").

Based on the results collected in Tables $2-5$, we have the following observations and remarks:

(i) The two-body charmless hadronic $B_{c} \rightarrow M_{1} M_{2}$ decays can be classified into two categories: the strangeness-conserving $(|\Delta S|=0)$ and the strangeness-changing $(|\Delta S|=1)$ processes. From the numerical results listed in Table 2, one can see that the branching rations of $|\Delta S|=0$ channels are generally much larger than those of $|\Delta S|=1$ ones. This is due to the large hierarchical structure between the two CKM matrix elements $V_{u d}$ and $V_{u s}$, $\left|V_{u d} / V_{u s}\right|^{2} \sim 19$

(ii) In scheme I, the branching ratios obtained with $\mathrm{W}$ I and W-III are very close, which vary in the ranges of $10^{-10}$ to $10^{-8}$, being larger than the corresponding ones obtained with W-II. This is consistent with the wave functions; the sharp of W-III is very close to $\delta$ function as shown in Figure 2. In scheme II, the annihilation contributions are enhanced when we adopt the IR finite gluon propagator, and the branching ratios are not sensitive to the choice of wave function for $B_{c}$ meson.

(iii) Among these charmless $B_{c}$ decays, only several decays modes, such as $B_{c}^{-} \rightarrow K^{-} K^{0}, K^{*-} K^{0}, K^{-} K^{* 0}, \pi^{-} \omega$, and $\rho^{-} \eta^{(\prime)}$, have relatively large branching ratios, being around $\mathcal{O}\left(10^{-7}\right)$ in scheme II. All of these channels belong to the $|\Delta S|=0$ transitions that are CKM favored. It is found that branching ratio for $B_{c}^{-} \rightarrow \pi^{-} \omega$ decay is relatively large among $B_{c}$ decays into $P P(V)$ final states and $\operatorname{Br}\left(B_{c}^{-} \rightarrow \pi^{-} \omega\right)=12.8 \times$ $10^{-8}$, which is promisingly detected by experiments at 
the running Large Hadron Collider and forthcoming SuperKEKB.

(iv) For $B_{c}^{-} \rightarrow \pi^{-} \pi^{0}, \pi^{-} \rho^{0}$, and $\rho^{-} \pi^{0}$ decays, on the other hand, since $\left|\pi^{0}\right\rangle,\left|\rho^{0}\right\rangle=(|\bar{u} u\rangle-|\bar{d} d\rangle) / \sqrt{2}$, the contributions from $\bar{u} u$ and $\bar{d} d$ components of the neutral mesons cancel each other exactly or almost, resulting in (approximate) zero branching ratios of these three channels. For $B_{c}^{-} \rightarrow \pi^{-} \omega, \pi^{-} \eta^{(\prime)}$, and $\rho^{-} \eta^{(\prime)}$ decays, due to the flavor decomposition $|\omega\rangle,\left|\eta_{q}\right\rangle=(|\bar{u} u\rangle+|\bar{d} d\rangle) / \sqrt{2}$, the interference between the two flavor components $\bar{u} u$ and $\bar{d} d$ of the neutral mesons is constructive, resulting in larger branching ratios. Taking into account the fact that $f_{\omega}>f_{\eta}>$ $f_{\eta^{\prime}}$, one can easily understand the pattern of their branching ratios. In particular, the decay modes $\pi^{-}\left(\rho^{-}\right) \eta$ and $\pi^{-}\left(\rho^{-}\right) \eta^{\prime}$ have similar branching ratios, because only the $\left|\eta_{q}\right\rangle$ term is involved in the decay amplitudes.

(v) For $B_{c}^{-} \rightarrow K^{(*)-} \eta^{(\prime)}$ decays, the obtained branching ratios show a rather different pattern, $\operatorname{Br}\left(K^{(*)-} \eta^{\prime}\right) \gg$ $\operatorname{Br}\left(K^{(*)-} \eta\right)$, from that of $\operatorname{Br}\left(\pi^{-}\left(\rho^{-}\right) \eta^{\prime}\right) \sim \operatorname{Br}\left(\pi^{-}\left(\rho^{-}\right) \eta\right)$. It is also observed that $\operatorname{Br}\left(K^{(*)-} \eta^{\prime}\right)$ is much larger than $\operatorname{Br}\left(K^{(*)-} \pi^{0}\right)$, while $\operatorname{Br}\left(K^{(*)-} \eta\right)$ is suppressed rather than enhanced compared to that of the $\pi^{0}$ mode. To understand the enhancement and suppression patterns, we should note that both the $\left|\eta_{q}\right\rangle$ and $\left|\eta_{s}\right\rangle$ terms contribute to these $|\Delta S|=1$ transitions, but with an opposite sign between them for the $\eta$ and $\eta^{\prime}$ final states, which is due to the fact that $f_{\eta^{(\prime)}}^{q}>0$, $f_{\eta^{\prime}}^{s}>0$, while $f_{\eta}^{s}<0$. This results in a destructive interference for $\eta$, but a constructive interference for the $\eta^{\prime}$ modes. Similar patterns have already been observed in the $B \rightarrow K \eta^{(\prime)}$ and $B \rightarrow K^{*} \eta^{(\prime)}$ decays [51].

(vi) As discussed in [26], several relations among the charmless $B_{c}$ decay channels hold in the limit of exact $\mathrm{SU}(3)$ flavor symmetry. For $B_{c} \rightarrow P P$ decays, for example, one of such relations reads

$$
\begin{aligned}
\mathscr{A}\left(B_{c}^{-} \longrightarrow \bar{K}^{0} \pi^{-}\right) & =\sqrt{2} \mathscr{A}\left(B_{c}^{-} \longrightarrow K^{-} \pi^{0}\right) \\
& =\hat{\lambda} \mathscr{A}\left(B_{c}^{-} \longrightarrow K^{-} K^{0}\right),
\end{aligned}
$$

with the Cabibbo-suppressing factor $\hat{\lambda}=V_{u s} / V_{u d}$. Similar relations could also be found for $B_{c} \rightarrow$ $P V$ decays, with the replacements $\pi \rightarrow \rho$ and/or $K \rightarrow K^{*}$. We find that the first equality holds exactly in both scheme I and scheme II, because the exact isospin symmetry is assumed in our calculation. The second equality is, however, violated by the differences between decay constants and light-quark masses, which account for the SU(3) breaking effect.

(vii) As a comparison, the pQCD predictions [25] are shown in the last column of Table 2. On the whole, the central values for the branching fractions obtained in this paper are smaller than those obtained in the pQCD approach. The different choice of the renormalization scale may be the main reason leading to these discrepancies. There are two points to notice about the results: (1) As mentioned earlier, for $B_{c}^{-} \rightarrow$ $\pi^{-} \rho^{0}, \rho^{-} \pi^{0}$ decays, their branching ratios are zero or almost zero in our result. But their branching ratios are not very small in the pQCD method, as shown in Table 2. (2) For $B_{c}^{-} \rightarrow K^{*-} K^{0}, K^{-} K^{* 0}$ decays, their branching ratios are almost the same in our results. And in the pQCD approach, $\operatorname{Br}\left(B_{c}^{-} \rightarrow\right.$ $\left.K^{-} K^{* 0}\right) \approx 5 \times \operatorname{Br}\left(B_{c}^{-} \rightarrow K^{*-} K^{0}\right)$. The possible causes of the two differences are the same: In the pQCD approach, they use the LCDAs in the form of Gegenbauer polynomials which contain the SU(3) breaking effect.

(viii) As the relevant CKM parameters have been measured quite precisely, the theoretical errors introduced by the CKM parameters are small. The uncertainty due to the variation of the dynamical gluon mass in scheme II is also found to be negligible. The main uncertainties are due to the variation of the renormalization scale, as well as the mixing parameters for $\eta$ and $\eta^{\prime}$ final states. The large scale dependence of the branching ratios is understandable, because only the leading order term in $\alpha_{s}$ is taken into account in our calculation. Furthermore, the different choices of the renormalization scale also account for the main differences among our results and the ones presented in $[25,26]$.

Finally, we would like to point out that it is hard to estimate the systematical uncertainties coming from the hypothesis underlying our calculations, such as the onegluon approximation for the annihilation mechanism, the use of asymptotic distribution amplitudes, and the neglect of $1 / m_{b}$-suppressed power corrections.

\section{Summary}

Being the lowest-lying bound state of two heavy quarks with different flavors, the $B_{c}$ meson is an ideal system to study weak decays of heavy mesons. In this paper, we have carried out a detailed study of two-body charmless hadronic $B_{c}$ decays, which can proceed only via the weak annihilation diagrams within the SM and are, therefore, very suitable for further improving our understanding of the annihilation mechanism, the size of which is currently an important issue in $B$ physics. Explicitly, we have adopted two different schemes to deal with these decays: scheme I is similar to the usual method adopted in the QCDF approach, while scheme II is based on the infrared behavior of gluon propagator and running coupling. For comparison, we adopt three different kinds of distribution function for $B_{c}$ meson in our calculation. It is found that the strength of annihilation contributions predicted in scheme II is enhanced compared to that obtained in scheme I. The branching ratios are 
TABLE 1: Relevant input parameters entering our numerical analysis. Details on the extraction of decay constants of vector mesons could be found in $[52,53]$.

\begin{tabular}{lr}
\hline$G_{F}=1.1663787 \times 10^{-5} \mathrm{GeV}^{-2}[54]$ & $f_{B_{c}}=487 \pm 5 \mathrm{MeV}[55]$ \\
$m_{b}=4.8 \pm 0.1 \mathrm{GeV}[54]$ & $\tau_{B_{c}}=0.452 \pm 0.033 \mathrm{ps}[54]$ \\
$m_{c}=1.5 \pm 0.1 \mathrm{GeV}[54]$ & $f_{\pi}=130.41 \pm 0.20 \mathrm{MeV}[54]$ \\
$\bar{m}_{s}(2 \mathrm{GeV})=95 \pm 5 \mathrm{MeV}[54]$ & $f_{K}=156.1 \pm 0.8 \mathrm{MeV}[54]$ \\
$\bar{m}_{s} / \bar{m}_{q}=27.5 \pm 1.0[54]$ & $f_{\rho}=215 \pm 6 \mathrm{MeV}[52,53]$ \\
$f_{\rho}^{\perp}(2 \mathrm{GeV}) / f_{\rho}=0.70 \pm 0.04[52,53]$ & $f_{K^{*}}=209 \pm 7 \mathrm{MeV}[52,53]$ \\
$f_{K^{*}}^{\perp}(2 \mathrm{GeV}) / f_{K^{*}}=0.73 \pm 0.04[52,53]$ & $f_{\omega}=188 \pm 10 \mathrm{MeV}[52,53]$ \\
$f_{\omega}^{\perp}(2 \mathrm{GeV}) / f_{\omega}=0.70 \pm 0.10[52,53]$ & $f_{\phi}=229 \pm 3 \mathrm{MeV}[52,53]$ \\
$f_{\phi}^{\perp}(2 \mathrm{GeV}) / f_{\phi}=0.750 \pm 0.020[52,53]$ & $m_{g}=0.49 \pm 0.03 \mathrm{GeV}[30,31]$ \\
\hline
\end{tabular}

TABLE 2: The CP-averaged branching ratios (in units of $10^{-8}$ for $|\Delta S|=0$ and $10^{-9}$ for $|\Delta S|=1$ transitions) of $B_{c} \rightarrow P P$ (upper) and $B_{c} \rightarrow P V$ (lower) decays based on the two schemes and three kinds of $B_{c}$ meson distribution function. The pQCD predictions [25] are also shown in the last column.

\begin{tabular}{|c|c|c|c|c|c|c|c|c|}
\hline \multirow{2}{*}{ Decay modes } & \multirow{2}{*}{ Cases } & \multicolumn{2}{|c|}{ Wave-I } & \multicolumn{2}{|c|}{ Wave-II } & \multicolumn{2}{|c|}{ Wave-III } & \multirow{2}{*}{$\mathrm{pQCD}$} \\
\hline & & S-I & S-II & S-I & S-II & S-I & S-II & \\
\hline$B_{c}^{-} \rightarrow \pi^{-} \pi^{0}$ & $|\Delta S|=0$ & 0 & 0 & 0 & 0 & 0 & 0 & 0 \\
\hline$B_{c}^{-} \rightarrow \pi^{-} \eta$ & $|\Delta S|=0$ & 2.82 & 5.50 & 1.30 & 4.87 & 3.00 & 5.61 & 22.8 \\
\hline$B_{c}^{-} \rightarrow \pi^{-} \eta^{\prime}$ & $|\Delta S|=0$ & 1.86 & 3.63 & 0.86 & 3.21 & 1.98 & 3.70 & 15.3 \\
\hline$B_{c}^{-} \rightarrow K^{-} K^{0}$ & $|\Delta S|=0$ & 4.69 & 9.15 & 2.18 & 9.11 & 5.01 & 9.25 & 24.0 \\
\hline$B_{c}^{-} \rightarrow K^{-} \pi^{0}$ & $|\Delta S|=1$ & 0.92 & 1.79 & 0.43 & 1.86 & 0.98 & 1.81 & $2.0 \times 10^{1}$ \\
\hline$B_{c}^{-} \rightarrow \bar{K}^{0} \pi^{-}$ & $|\Delta S|=1$ & 1.84 & 3.59 & 0.86 & 3.72 & 1.97 & 3.61 & $4.0 \times 10^{1}$ \\
\hline$B_{c}^{-} \rightarrow K^{-} \eta$ & $|\Delta S|=1$ & 0.17 & 0.33 & 0.08 & 0.45 & 0.18 & 0.33 & $0.6 \times 10^{1}$ \\
\hline$B_{c}^{-} \rightarrow K^{-} \eta^{\prime}$ & $|\Delta S|=1$ & 3.85 & 7.52 & 1.79 & 7.28 & 4.11 & 7.62 & $5.7 \times 10^{1}$ \\
\hline$B_{c}^{-} \rightarrow \pi^{-} \rho^{0}$ & $|\Delta S|=0$ & 0 & 0 & 0 & 0.02 & 0 & $\sim 0$ & $1.7 \times 10^{1}$ \\
\hline$B_{c}^{-} \rightarrow \rho^{-} \pi^{0}$ & $|\Delta S|=0$ & 0 & 0 & 0 & 0.02 & 0 & $\sim 0$ & $0.5 \times 10^{1}$ \\
\hline$B_{c}^{-} \rightarrow \pi^{-} \omega$ & $|\Delta S|=0$ & 6.19 & 12.4 & 2.63 & 10.2 & 6.59 & 12.8 & $5.8 \times 10^{1}$ \\
\hline$B_{c}^{-} \rightarrow \rho^{-} \eta$ & $|\Delta S|=0$ & 5.34 & 10.6 & 2.32 & 7.43 & 5.67 & 11.0 & $5.4 \times 10^{1}$ \\
\hline$B_{c}^{-} \rightarrow \rho^{-} \eta^{\prime}$ & $|\Delta S|=0$ & 3.52 & 7.00 & 1.53 & 4.90 & 3.74 & 7.26 & $3.6 \times 10^{1}$ \\
\hline$B_{c}^{-} \rightarrow K^{*-} K^{0}$ & $|\Delta S|=0$ & 5.46 & 11.0 & 2.31 & 8.81 & 5.82 & 11.3 & $1.8 \times 10^{1}$ \\
\hline$B_{c}^{-} \rightarrow K^{-} K^{* 0}$ & $|\Delta S|=0$ & 5.46 & 11.0 & 2.31 & 9.94 & 5.82 & 11.3 & $10.0 \times 10^{1}$ \\
\hline$B_{c}^{-} \rightarrow K^{-} \rho^{0}$ & $|\Delta S|=1$ & 1.53 & 3.07 & 0.65 & 2.30 & 1.63 & 3.17 & $3.1 \times 10^{1}$ \\
\hline$B_{c}^{-} \rightarrow \bar{K}^{0} \rho^{-}$ & $|\Delta S|=1$ & 3.06 & 6.14 & 1.31 & 4.61 & 3.26 & 6.33 & $6.1 \times 10^{1}$ \\
\hline$B_{c}^{-} \rightarrow K^{*-} \pi^{0}$ & $|\Delta S|=1$ & 1.04 & 2.09 & 0.44 & 1.98 & 1.11 & 2.14 & $1.6 \times 10^{1}$ \\
\hline$B_{c}^{-} \rightarrow \bar{K}^{* 0} \pi^{-}$ & $|\Delta S|=1$ & 2.07 & 4.19 & 0.87 & 3.95 & 2.21 & 4.28 & $3.3 \times 10^{1}$ \\
\hline$B_{c}^{-} \rightarrow K^{-} \omega$ & $|\Delta S|=1$ & 1.17 & 2.35 & 0.50 & 1.77 & 1.25 & 2.42 & $2.3 \times 10^{1}$ \\
\hline$B_{c}^{-} \rightarrow K^{*-} \eta$ & $|\Delta S|=1$ & 0.15 & 0.32 & 0.06 & 0.29 & 0.16 & 0.32 & $0.9 \times 10^{1}$ \\
\hline$B_{c}^{-} \rightarrow K^{*-} \eta^{\prime}$ & $|\Delta S|=1$ & 4.58 & 9.22 & 1.95 & 7.41 & 4.88 & 9.47 & $3.8 \times 10^{1}$ \\
\hline$B_{c}^{-} \rightarrow \phi K^{-}$ & $|\Delta S|=1$ & 3.55 & 7.18 & 1.49 & 7.02 & 3.78 & 7.33 & $5.6 \times 10^{1}$ \\
\hline
\end{tabular}

not sensitive to the choice of wave function for $B_{c}$ meson in scheme II. However, the predicted branching ratios are inconsistent with the corresponding ones obtained in the pQCD approach [25].

The large discrepancies among these theoretical predictions make it very necessary to make more detailed studies of these kinds of $B_{c}$ decays, especially from the experimental side. It is interesting to note that the $\mathrm{LHCb}$ experiment has the potential to observe the decays with a branching ratio of $10^{-7}$, which will certainly provide substantial information on these charmless $B_{c}$ decays and deepen our understanding of the annihilation mechanisms.

\section{Appendix}

\section{A. Decay Amplitudes in the QCDF Approach}

Starting with (3) and adopting the standard phase convention for the flavor wave functions of light and heavy mesons $[21,51,56]$, one can easily write down the decay amplitude for 
TABLE 3: The CP-averaged branching ratios and theoretical errors (in units of $10^{-8}$ for $|\Delta S|=0$ and $10^{-9}$ for $|\Delta S|=1$ transitions) of $B_{c} \rightarrow P P$ (upper) and $B_{c} \rightarrow P V$ (lower) decays based on W-I. The theoretical errors correspond to the uncertainties referred to as "CKM," "hadronic," "scale," and " $m_{g}$ " defined in the text.

\begin{tabular}{|c|c|c|c|}
\hline Decay modes & Cases & Scheme I & Scheme II \\
\hline$B_{c}^{-} \rightarrow \pi^{-} \pi^{0}$ & $|\Delta S|=0$ & 0 & 0 \\
\hline$B_{c}^{-} \rightarrow \pi^{-} \eta$ & $|\Delta S|=0$ & $2.82_{-0.20-1.94-2.03}^{+0.13+2.68+7.64}$ & $5.50_{-0.39-3.77-3.16-0.12}^{+0.26+5.17+6.15+0.13}$ \\
\hline$B_{c}^{-} \rightarrow \pi^{-} \eta^{\prime}$ & $|\Delta S|=0$ & $1.86_{-0.14-1.30-1.34}^{+0.08+1.04}$ & $3.63_{-0.26-2.54-2.08-0.08}^{+0.16+3.48+4.08}$ \\
\hline$B_{c}^{-} \rightarrow K^{-} K^{0}$ & $|\Delta S|=0$ & $4.69_{-0.33-0.19-3.34}^{+0.22+1.30-19.34}$ & $9.15_{-0.65-0.37-5.15-0.20}^{+0.26-2.54-0.38+70-0.08}$ \\
\hline$B_{c}^{-} \rightarrow K^{-} \pi^{0}$ & $|\Delta S|=1$ & $0.92_{-0.07-0.03-0.03+2.37}^{+0.05}$ & $1.79_{-0.13-0.006-10.06+1.87+0.04}^{+0.00}$ \\
\hline$B_{c}^{-} \rightarrow \bar{K}^{0} \pi^{-}$ & $|\Delta S|=1$ & $1.84_{-0.14-0.008-1.31}^{+0.09+0.06+.03}$ & $3.59_{-0.27-0.12-2.01-0.08}^{+0.19+0.12+3.72+0.08}$ \\
\hline$B_{c}^{-} \rightarrow K^{-} \eta$ & $|\Delta S|=1$ & $0.17_{-0.01-0.018-0.42}^{+0.14-0.018-1.31}$ & $0.33_{-0.02-0.035-0.017-0.00}^{+0.02+0.12-0.01-0.08}$ \\
\hline$B_{c}^{-} \rightarrow K^{-} \eta^{\prime}$ & $|\Delta S|=1$ & $3.85_{-0.28-0.62-2.75}^{+0.20+0.18+10.16}$ & $7.52_{-0.56-1.21-4.025+1.029-0.17}^{+0.017-0.00}$ \\
\hline$B_{c}^{-} \rightarrow \pi^{-} \rho^{0}$ & $|\Delta S|=0$ & 0 & 0 \\
\hline$B_{c}^{-} \rightarrow \rho^{-} \pi^{0}$ & $|\Delta S|=0$ & 0 & 0 \\
\hline$B_{c}^{-} \rightarrow \pi^{-} \omega$ & $|\Delta S|=0$ & $6.19_{-0.44-0.84-4.61}^{+0.29+0.92+19.23}$ & $12.4_{-0.8-1.7-7.6-0.3}^{+0.6+2.0+16.6+0.3}$ \\
\hline$B_{c}^{-} \rightarrow \rho^{-} \eta$ & $|\Delta S|=0$ & $5.34_{-0.38-0.74-3.92 .98}^{+0.25}$ & $10.6_{-0.7-1.8-6.5-0.2}^{+0.5+2.1+14+0.3}$ \\
\hline$B_{c}^{-} \rightarrow \rho^{-} \eta^{\prime}$ & $|\Delta S|=0$ & $3.52_{-0.25-0.164-2.63}^{+0.160+10.99}$ & $7.00_{-0.50-1.28-4.429-0.16}^{+0.33+1.48+9.41+0.16}$ \\
\hline$B^{-} \rightarrow K^{*-} K^{0}$ & $|\Delta S|=0$ & $5.46_{-0.29+0.58+16.95}^{+0.25}$ & $11.0_{-0.5+1.2+14.6+0.3}^{+0.160}$ \\
\hline$B_{c}^{-} \rightarrow K^{-} K^{* 0}$ & $|\Delta S|=0$ & $5.46_{-0.39-0.54-4.07}^{+0.25+0.58+16.94}$ & $11.0_{-0.8-1.1-6.7-0.3}^{+0.8+1.12+14.6 .3}$ \\
\hline$B_{c}^{-} \rightarrow K^{-} \rho^{0}$ & $|\Delta S|=1$ & $1.53_{-0.11-0.013-1.14}^{+0.08+0.15+4.77}$ & $3.07_{-0.23-0.27-1.1 .108-0.0 .08}^{+0.10+10+0.08}$ \\
\hline$B_{c}^{-} \rightarrow \bar{K}^{0} \rho^{-}$ & $|\Delta S|=1$ & $3.06_{-0.22-0.026+9.53}^{+0.16+0.28}$ & $6.14_{-0.35-0.59+8.21+0.15}^{+0.32+0.59+0.14}$ \\
\hline$B_{c}^{-} \rightarrow K^{*-} \pi^{0}$ & $|\Delta S|=1$ & $1.04_{-0.08-0.00-0.078}^{+0.22-0.26-2.28}$ & $2.09_{-0.15-0.19-1.27+0.05}^{+0.11+0.22+2.78+0.14}$ \\
\hline$B_{c}^{c} \rightarrow \bar{K}^{* 0} \pi^{-}$ & $|\Delta S|=1$ & $2.07_{-0.05-0.19-1.54}^{+0.08-11+.0 .21+6.78}$ & $4.19_{-0.31-0.40-2.56-0.10}^{+0.21+0.19+2.545+0.03}$ \\
\hline$B_{c}^{-} \rightarrow K^{-} \omega$ & $|\Delta S|=1$ & $1.17_{-0.08-0.16-0.87}^{+0.06+0.19+3}$ & $2.35_{-0.17-0.34-1.144-0.06}^{+0.05}$ \\
\hline$B_{c}^{-} \rightarrow K^{*-} \eta$ & $|\Delta S|=1$ & $0.15_{-0.01-0.08-0.11}^{+0.01+0.12-0.87}$ & $0.32_{-0.03-0.19-0.20-0.01}^{+0.01+0.27+0.41+0.00}$ \\
\hline$B_{c}^{-} \rightarrow K^{*-} \eta^{\prime}$ & $|\Delta S|=1$ & $4.58^{-0.24+0.37+14.23}$ & $9.22^{-0.47+0.73+12.27+0.22}$ \\
\hline$B_{c}^{-} \rightarrow \phi K^{-}$ & $|\Delta S|=1$ & $3.55^{-0.18+0.21+11.03}$ & $7.18^{+0.37+0.45+9.51+0.18}$ \\
\hline
\end{tabular}

a given decay mode. Firstly, there are eight charmless $B_{c} \rightarrow$ $P P$ decays with the corresponding amplitude given, respectively, as (the exact isospin symmetry is assumed):

$$
\begin{aligned}
& \mathscr{A}\left(B_{c}^{-} \longrightarrow \pi^{-} \pi^{0}\right)=\frac{G_{F}}{2} \\
& \cdot V_{c b} V_{u d}^{*} f_{B_{c}} f_{\pi^{-}} f_{\pi^{0}}\left[b_{2}\left(\pi^{0}, \pi^{-}\right)-b_{2}\left(\pi^{-}, \pi^{0}\right)\right] \\
& =0 \\
& \mathscr{A}\left(B_{c}^{-} \longrightarrow \pi^{-} \eta^{(\prime)}\right)=\frac{G_{F}}{2} \\
& \cdot V_{c b} V_{u d}^{*} f_{B_{c}} f_{\pi^{-}} f_{\eta^{(\prime)}}^{q}\left[b_{2}\left(\pi^{-}, \eta^{(\prime)}\right)+b_{2}\left(\eta^{(\prime)}, \pi^{-}\right)\right], \\
& \mathscr{A}\left(B_{c}^{-} \longrightarrow K^{-} K^{0}\right)=\frac{G_{F}}{\sqrt{2}} \\
& \cdot V_{c b} V_{u d}^{*} f_{B_{c}} f_{K^{-}} f_{K^{0}} b_{2}\left(K^{-}, K^{0}\right), \\
& \mathscr{A}\left(B_{c}^{-} \longrightarrow K^{-} \pi^{0}\right)=\frac{G_{F}}{2} \\
& \cdot V_{c b} V_{u s}^{*} f_{B_{c}} f_{K^{-}} f_{\pi^{0}} b_{2}\left(\pi^{0}, K^{-}\right), \\
& \mathscr{A}\left(B_{c}^{-} \longrightarrow \bar{K}^{0} \pi^{-}\right)=\frac{G_{F}}{\sqrt{2}} \\
& \cdot V_{c b} V_{u s}^{*} f_{B_{c}} f_{K^{0}} f_{\pi^{-}} b_{2}\left(\pi^{-}, \bar{K}^{0}\right),
\end{aligned}
$$

$$
\begin{aligned}
\mathscr{A} & \left(B_{c}^{-} \longrightarrow K^{-} \eta^{(\prime)}\right)=\frac{G_{F}}{2} \\
& \cdot V_{c b} V_{u s}^{*} f_{B_{c}} f_{K^{-}}\left[f_{\eta^{(\prime)}}^{q} b_{2}\left(\eta^{(\prime)}, K^{-}\right)\right. \\
& \left.+\sqrt{2} f_{\eta^{(\prime)}}^{s} b_{2}\left(K^{-}, \eta^{(\prime)}\right)\right] .
\end{aligned}
$$

The decay amplitudes for the 15 charmless $P V$ modes can be written, respectively, as:

$$
\begin{aligned}
& \mathscr{A}\left(B_{c}^{-} \longrightarrow \pi^{-} \rho^{0}\right)=\frac{G_{F}}{2} \\
& \cdot V_{c b} V_{u d}^{*} f_{B_{c}} f_{\pi^{-}} f_{\rho^{0}}\left[b_{2}\left(\rho^{0}, \pi^{-}\right)-b_{2}\left(\pi^{-}, \rho^{0}\right)\right], \\
& \mathscr{A}\left(B_{c}^{-} \longrightarrow \pi^{-} \omega\right)=\frac{G_{F}}{2} V_{c b} V_{u d}^{*} f_{B_{c}} f_{\pi^{-}} f_{\omega}\left[b_{2}\left(\omega, \pi^{-}\right)\right. \\
& \left.\quad+b_{2}\left(\pi^{-}, \omega\right)\right], \\
& \mathscr{A}\left(B_{c}^{-} \longrightarrow K^{*-} K^{0}\right)=\frac{G_{F}}{\sqrt{2}} \\
& \quad \cdot V_{c b} V_{u d}^{*} f_{B_{c}} f_{K^{*-}} f_{K^{0}} b_{2}\left(K^{*-}, K^{0}\right), \\
& \mathscr{A}\left(B_{c}^{-} \longrightarrow K^{-} \rho^{0}\right)=\frac{G_{F}}{2} \\
& \quad \cdot V_{c b} V_{u s}^{*} f_{B_{c}} f_{K^{-}} f_{\rho^{0}} b_{2}\left(\rho^{0}, K^{-}\right),
\end{aligned}
$$


TABLE 4: The CP-averaged branching ratios and theoretical errors (in units of $10^{-8}$ for $|\Delta S|=0$ and $10^{-9}$ for $|\Delta S|=1$ transitions) of $B_{c} \rightarrow P P$ (upper) and $B_{c} \rightarrow P V$ (lower) decays based on W-II. The theoretical errors correspond to the uncertainties referred to as "CKM," "hadronic," "scale," and " $m_{g}$ " defined in the text.

\begin{tabular}{|c|c|c|c|}
\hline Decay modes & Cases & Scheme I & Scheme II \\
\hline$B_{c}^{-} \rightarrow \pi^{-} \pi^{0}$ & $|\Delta S|=0$ & 0 & 0 \\
\hline$B_{c}^{-} \rightarrow \pi^{-} \eta$ & $|\Delta S|=0$ & $1.30_{-0.09-0.92-0.93}^{+0.06+1.41+3.48}$ & $4.87_{-0.35-5.56-2.55-0.30}^{+0.22+11.31+4.16+0.39}$ \\
\hline$B_{c}^{-} \rightarrow \pi^{-} \eta^{\prime}$ & $|\Delta S|=0$ & $0.86_{-0.06-0.04+0.032 .29}^{+0.02}$ & $3.21_{-0.23-3.71-1.68-0.20}^{+0.15+7.75-0.26}$ \\
\hline$B_{c}^{-} \rightarrow K^{-} K^{0}$ & $|\Delta S|=0$ & $\begin{array}{l}2.18_{-0.15-0.08-1.55}^{+0.00+0.09+56} \\
\end{array}$ & $9.11_{-0.64-0.38+0.36-0.01+0.82}^{+0.63}$ \\
\hline $\overrightarrow{B_{c}^{-} \rightarrow K^{-} \pi^{0}}$ & $|\Delta S|=1$ & $0.43_{-0.03-0.01-0.30}^{+0.02+0.01 .09}$ & $1.86_{-0.14-0.06-0.093-0.13}^{+0.13}$ \\
\hline$B_{c}^{-} \rightarrow \bar{K}^{0} \pi^{-}$ & $|\Delta S|=1$ & $0.86_{-0.06-0.03-0.061}^{+0.04+0.02+17}$ & 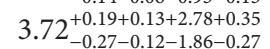 \\
\hline$B_{c}^{-} \rightarrow K^{-} \eta$ & $|\Delta S|=1$ & $0.08_{-0.00-0.08-0.05}^{+0.00-0.01+0.019}$ & $0.45_{-0.02+1.70+0.27+0.05}^{+0.02}$ \\
\hline$B_{c}^{-} \rightarrow K^{-} \eta^{\prime}$ & $|\Delta S|=1$ & $1.79_{-0.13-0.31-1.27}^{+0.09+0.035+4.05}$ & $7.28_{-0.35-2.069-3.77+0.65}^{+0.032-0.49}$ \\
\hline$B_{c}^{-} \rightarrow \pi^{-} \rho^{0}$ & $|\Delta S|=0$ & 0 & $0.02_{-0.000}^{+0.00000-0.01+0.02+0.00}$ \\
\hline$B_{c}^{-} \rightarrow \rho^{-} \pi^{0}$ & $|\Delta S|=0$ & 0 & $0.02_{-0.000-0.00-0.01-0.02}^{+0.000}$ \\
\hline$B_{c}^{-} \rightarrow \pi^{-} \omega$ & $|\Delta S|=0$ & $2.63_{-0.18-0.34-1.96}^{+0.12+0.37+8.25}$ & $10.2_{-0.7-1.9-6.5-1.1}^{+0.5+2.3+12.0 .02}$ \\
\hline$B_{c}^{-} \rightarrow \rho^{-} \eta$ & $|\Delta S|=0$ & 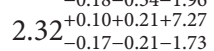 & $7.43_{-0.52-5.07-4.3447-0.57}^{+0.35+7.06+1.23}$ \\
\hline$B_{c}^{-} \rightarrow \rho^{-} \eta^{\prime}$ & $|\Delta S|=0$ & $1.53_{-0.07+0.15+4.79}^{+0.07+216}$ & $4.90_{-0.23+4.75+6.18+0.15}^{+0.23}$ \\
\hline$B_{c}^{-} \rightarrow K^{*-} K^{0}$ & $|\Delta S|=0$ & $2.31^{+0.11+0.24+7.24}$ & $8.81_{-0.42+1.17+2.27+0.77}^{+0.07}$ \\
\hline$B_{c}^{-} \rightarrow K^{-} K^{* 0}$ & $|\Delta S|=0$ & $2.31_{-0.16-0.22-1.72}^{+0.11+0.24+7.22}$ & $9.94_{-0.46+1.37+9.66+0.00}^{+0.00}$ \\
\hline$B_{c}^{-} \rightarrow K^{-} \rho^{0}$ & $|\Delta S|=1$ & $0.65_{-0.04+0.006-0.22-1.06}^{+0.06}$ & $2.30^{+0.12+1.23-0.27+2.889+1.60}$ \\
\hline$B_{c}^{-} \rightarrow \bar{K}^{0} \rho^{-}$ & $|\Delta S|=1$ & $1.31^{-0.007-0.07+0.12+4.11}$ & \\
\hline $\begin{array}{l}B_{c} \rightarrow \kappa \rho \\
D^{*-} 0^{*}\end{array}$ & & & $\begin{array}{r}4.6 I_{-0.34-0.50-2.76-0.24}+0.10+0.26+2.34+0.08\end{array}$ \\
\hline$B_{c}^{-} \rightarrow K^{*-} \pi^{0}$ & $|\Delta S|=1$ & $0.44_{-0.04-0.04-0.33}^{+0.02+0.04+1.36}$ & $1.98_{-0.15-0.24-1.17-0.34}^{+0.10+0.26+2.34+0.08}$ \\
\hline$B_{c}^{-} \rightarrow \bar{K}^{* 0} \pi^{-}$ & $|\Delta S|=1$ & $0.87_{-0.06-0.008-0.05}^{+0.05+2.03}$ & $3.95_{-0.29-0.47-2.34+0.064}^{+0.20}$ \\
\hline$B_{c}^{-} \rightarrow K^{-} \omega$ & $|\Delta S|=1$ & $0.50_{-0.04-0.07+0.037}^{+0.008+0.57}$ & $1.77_{-0.13-0.033-1.06-0.09}^{+0.09+0.19+0.14}$ \\
\hline$B_{c}^{-} \rightarrow K^{*-} \eta$ & $|\Delta S|=1$ & $0.06_{-0.00-0.02+0.0040 .04}^{+0.040 .03}$ & $0.29_{-0.02-0.32-0.017-0.02}^{+0.01-0.33+1.06-0.09}$ \\
\hline$B_{c}^{-} \rightarrow K^{*-} \eta^{\prime}$ & $|\Delta S|=1$ & $1.95_{-0.10+0.17+6.10}^{+0.00-0.02}$ & $7.41_{-0.55-1.84-4.41-0.39}^{+0.39}$ \\
\hline$B_{c}^{-} \rightarrow \phi K^{-}$ & $|\Delta S|=1$ & $\begin{array}{r}-.15-0.17-1.46 \\
1.49_{-0.11-0.08+4.09-1.11}^{+0.05}\end{array}$ & 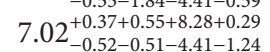 \\
\hline
\end{tabular}

$$
\begin{aligned}
& \mathscr{A}\left(B_{c}^{-} \longrightarrow \bar{K}^{0} \rho^{-}\right)=\frac{G_{F}}{\sqrt{2}} \\
& \cdot V_{c b} V_{u s}^{*} f_{B_{c}} f_{K^{0}} f_{\rho^{-}} b_{2}\left(\rho^{-}, \bar{K}^{0}\right), \\
& \mathscr{A}\left(B_{c}^{-} \longrightarrow K^{-} \omega\right)=\frac{G_{F}}{2} V_{c b} V_{u s}^{*} f_{B_{c}} f_{K^{-}} f_{\omega} b_{2}\left(\omega, K^{-}\right), \\
& \mathscr{A}\left(B_{c}^{-} \longrightarrow \rho^{-} \pi^{0}\right)=\frac{G_{F}}{2} \\
& \cdot V_{c b} V_{u d}^{*} f_{B_{c}} f_{\rho^{-}} f_{\pi^{0}}\left[b_{2}\left(\pi^{0}, \rho^{-}\right)-b_{2}\left(\rho^{-}, \pi^{0}\right)\right], \\
& \mathscr{A}\left(B_{c}^{-} \longrightarrow \rho^{-} \eta^{(\prime)}\right)=\frac{G_{F}}{2} \\
& \cdot V_{c b} V_{u d}^{*} f_{B_{c}} f_{\rho^{-}} f_{\eta^{(\prime)}}^{q}\left[b_{2}\left(\eta^{(\prime)}, \rho^{-}\right)+b_{2}\left(\rho^{-}, \eta^{(\prime)}\right)\right], \\
& \mathscr{A}\left(B_{c}^{-} \longrightarrow K^{-} K^{* 0}\right)=\frac{G_{F}}{\sqrt{2}} \\
& \cdot V_{c b} V_{u d}^{*} f_{B_{c}} f_{K^{-}} f_{K^{* 0}} b_{2}\left(K^{-}, K^{* 0}\right), \\
& \mathscr{A}\left(B_{c}^{-} \longrightarrow K^{*-} \pi^{0}\right)=\frac{G_{F}}{2} \\
& \cdot V_{c b} V_{u s}^{*} f_{B_{c}} f_{K^{*-}} f_{\pi^{0}} b_{2}\left(\pi^{0}, K^{*-}\right),
\end{aligned}
$$

$$
\begin{aligned}
& \mathscr{A}\left(B_{c}^{-} \longrightarrow \bar{K}^{* 0} \pi^{-}\right)=\frac{G_{F}}{\sqrt{2}} \\
& \cdot V_{c b} V_{u s}^{*} f_{B_{c}} f_{K^{* 0}} f_{\pi^{-}} b_{2}\left(\pi^{-}, \bar{K}^{* 0}\right) \\
& \mathscr{A}\left(B_{c}^{-} \longrightarrow K^{*-} \eta^{(\prime)}\right)=\frac{G_{F}}{2} \\
& \cdot V_{c b} V_{u s}^{*} f_{B_{c}} f_{K^{*-}}\left[f_{\eta^{(\prime)}}^{q} b_{2}\left(\eta^{(\prime)}, K^{*-}\right)\right. \\
& \left.+\sqrt{2} f_{\eta^{(\prime)}}^{s} b_{2}\left(K^{*-}, \eta^{(\prime)}\right)\right] \\
& \mathscr{A}\left(B_{c}^{-} \longrightarrow \phi K^{-}\right)=\frac{G_{F}}{\sqrt{2}} V_{c b} V_{u s}^{*} f_{B_{c}} f_{\phi} f_{K^{-}} b_{2}\left(K^{-}, \phi\right) .
\end{aligned}
$$

\section{B. Input Parameters}

To get the Wilson coefficients $C_{i}(\mu)$ at the lower scale $\mu=$ $m_{B_{c}} / 2$, we adopt the following input parameters [54]:

$$
\begin{aligned}
\alpha_{s}\left(M_{Z}\right) & =0.1185 \pm 0.0006, \\
\alpha\left(M_{Z}\right) & =\frac{1}{128} \\
\sin ^{2} \theta_{W} & =0.23
\end{aligned}
$$


TABLE 5: The CP-averaged branching ratios and theoretical errors (in units of $10^{-8}$ for $|\Delta S|=0$ and $10^{-9}$ for $|\Delta S|=1$ transitions) of $B_{c} \rightarrow P P$ (upper) and $B_{c} \rightarrow P V$ (lower) decays based on W-III. The theoretical errors correspond to the uncertainties referred to as "CKM," "hadronic," "scale," and " $m_{g}$ " defined in the text.

\begin{tabular}{|c|c|c|c|}
\hline Decay modes & Cases & Scheme I & Scheme II \\
\hline$B_{c}^{-} \rightarrow \pi^{-} \pi^{0}$ & $|\Delta S|=0$ & 0 & 0 \\
\hline$B_{c}^{-} \rightarrow \pi^{-} \eta$ & $|\Delta S|=0$ & $3.00_{-0.21-2.08-2.16}^{+0.14+2.91+8.13}$ & $5.61_{-0.40-5.10-3.24-0.08}^{+0.26+4.80+6.38+0.24}$ \\
\hline$B_{c}^{-} \rightarrow \pi^{-} \eta^{\prime}$ & $|\Delta S|=0$ & $1.98_{-0.14-0.09+1.96-5.36}^{-0.36}$ & $3.70_{-0.26-3.18-2.14-0.05}^{+0.17+2.15}$ \\
\hline$B_{c}^{-} \rightarrow K^{-} K^{0}$ & $|\Delta S|=0$ & $5.01_{-0.36-0.20-3.57}^{+0.23}$ & $9.25_{-0.66-0.37-5.25-0.13}^{+0.43+0.37}$ \\
\hline $\overrightarrow{B_{c}^{-} \rightarrow K^{-} \pi^{0}}$ & $|\Delta S|=1$ & $0.98_{-0.07-0.03-0.030}^{+0.052}$ & $1.81_{-0.14-0.06-1.09+02-0.04}^{+0.09}$ \\
\hline$B_{c}^{-} \rightarrow \bar{K}^{0} \pi^{-}$ & $|\Delta S|=1$ & $1.97_{-0.15-0.067-1.40}^{+0.10+0.04}$ & $3.61_{-0.26-0.12-0.03-0.03+0.08}^{+0.19+3}$ \\
\hline$B_{c}^{-} \rightarrow K^{-} \eta$ & $|\Delta S|=1$ & $0.18^{+0.01+0.39+0.43}$ & $0.33_{-0.02+0.63+0.31+0.01}^{+0.02}$ \\
\hline$B_{c}^{-} \rightarrow K^{-} \eta^{\prime}$ & $|\Delta S|=1$ & $\begin{array}{c}-0.01-0.19-0.12 \\
4.11_{-0.20-0.74+10.82}^{+0.22+2.93}\end{array}$ & $7.62_{-0.56-1.06-4.34-0.16}^{+0.029+0.34-0.19}$ \\
\hline$B_{c}^{-} \rightarrow \pi^{-} \rho^{0}$ & $|\Delta S|=0$ & 0 & $\begin{array}{c}-0.50-1.00-4.54-0.10 \\
\sim 0\end{array}$ \\
\hline$B_{c}^{-} \rightarrow \rho^{-} \pi^{0}$ & $|\Delta S|=0$ & 0 & $\sim 0$ \\
\hline$B_{c}^{-} \rightarrow \pi^{-} \omega$ & $|\Delta S|=0$ & $6.59_{-0.47-0.89-4.91}^{+0.31+0.99+20.46}$ & $12.8_{-0.9-1.8-7.8-0.3}^{+0.6+1.9+17.1+0.7}$ \\
\hline$B_{c}^{-} \rightarrow \rho^{-} \eta$ & $|\Delta S|=0$ & $5.67_{-0.40-0.82-0.23}^{+0.27+0.93+17.72}$ & $11.0_{-0.8-1.54-6.7-0.1}^{+0.5+1.8+0.6}$ \\
\hline$B_{c}^{-} \rightarrow \rho^{-} \eta^{\prime}$ & $|\Delta S|=0$ & $3.74_{-0.27-0.61-2.79}^{+0.17+0.67+1.67}$ & $7.26_{-0.52-1.10-4.30+45+0.40}^{+0.34}$ \\
\hline$B_{c}^{-} \rightarrow K^{*-} K^{0}$ & $|\Delta S|=0$ & $5.82_{-0.41-0.58-4.34}^{+0.27+0.62+18.04}$ & $11.3_{-0.8-1 .-6.15-0.6}^{+0.5+15}$ \\
\hline$B_{c}^{-} \rightarrow K^{-} K^{* 0}$ & $|\Delta S|=0$ & 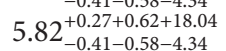 & $11.3_{-0.0-1.1-6.9-0.2}^{+0.0+1.1-6.15-1+2}$ \\
\hline$B_{c}^{-} \rightarrow K^{-} \rho^{0}$ & $|\Delta S|=1$ & $1.63_{-0.12-0.14-1.21}^{+0.09+0.16 .5 .7}$ & $3.17_{-0.24-0.28-1.29+4.24-0.06}^{+0.06}$ \\
\hline$B_{c}^{-} \rightarrow \bar{K}^{0} \rho^{-}$ & $|\Delta S|=1$ & $3.26^{+0.17+0.31+10.14}$ & $6.33^{-0.33+0.60+8.49+0.13}$ \\
\hline$B_{c}^{-} \rightarrow K^{*-} \pi^{0}$ & $|\Delta S|=1$ & $\begin{array}{c}-0.24-0.29-2.43 \\
1.11_{-0.05+0.11+3.42}^{+0.05+11-0.83}\end{array}$ & $2.14_{-0.11+0.22+2.86+0.05}^{+0.47-0.56-10}$ \\
\hline$B_{c}^{-} \rightarrow \bar{K}^{* 0} \pi^{-}$ & $|\Delta S|=1$ & $2.21_{-0.16-0.21-6.84}^{+0.09-0.11+0.83}$ & 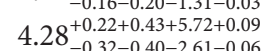 \\
\hline$B_{c}^{-} \rightarrow K^{-} \omega$ & $|\Delta S|=1$ & $1.25_{-0.09-0.18-0.93}^{+0.06+0.219-1.65}$ & $2.42_{-0.18-0.34-1.48-0.04}^{+0.32-0.40-2.61-0.06}$ \\
\hline$B_{c}^{-} \rightarrow K^{*-} \eta$ & $|\Delta S|=1$ & $\begin{array}{r}-0.09-0.18-0.93 \\
0.16_{-0.01-0.08-0.013}^{+0.01+0.12}\end{array}$ & 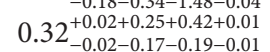 \\
\hline$B_{c}^{-} \rightarrow K^{*-} \eta^{\prime}$ & $|\Delta S|=1$ & $4.88_{-0.036-0.38+2.88}^{+0.01-0.08}$ & $9.47_{-0.49+0.77+7.26+0.20}^{+0.01}$ \\
\hline$B_{c}^{-} \rightarrow \phi K^{-}$ & $|\Delta S|=1$ & $3.78_{-0.28-0.22-2.81}^{+0.36-0.247-3.64}$ & $7.33_{-0.54-0.43-4.43+48-0.16}^{+0.038}$ \\
\hline
\end{tabular}

$$
\begin{aligned}
M_{Z} & =91.1876 \mathrm{GeV} \\
M_{W} & =80.385 \mathrm{GeV} \\
m_{t} & =173.21 \pm 0.87 \mathrm{GeV} .
\end{aligned}
$$

We also vary the renormalization scale $\mu$ in the region $\left[m_{B_{c}} / 4, m_{B_{c}}\right]$ to assess the scale uncertainty.

For the CKM matrix elements, we use the Wolfenstein parameterization [57] and keep terms up to $\mathcal{O}\left(\lambda^{4}\right)$ [35]:

$$
\begin{aligned}
V_{u d} & =1-\frac{1}{2} \lambda^{2}-\frac{1}{8} \lambda^{4}+\mathcal{O}\left(\lambda^{6}\right), \\
V_{u s} & =\lambda+\mathcal{O}\left(\lambda^{7}\right), \\
V_{c b} & =A \lambda^{2}+\mathcal{O}\left(\lambda^{8}\right),
\end{aligned}
$$

with the inputs $A=0.813_{-0.027}^{+0.015}$ and $\lambda=0.22551_{-0.00035}^{+0.00068}[58]$.

For the $\eta-\eta{ }^{\prime}$ system, we adopt the Feldmann-Kroll-Stech (FKS) mixing scheme defined in the quark-flavor basis [56], where the physical states $|\eta\rangle$ and $\left|\eta^{\prime}\right\rangle$ are related to the flavor states $\left|\eta_{q}\right\rangle=(|\bar{u} u\rangle+|\bar{d} d\rangle) / \sqrt{2}$ and $\left|\eta_{s}\right\rangle=|s \bar{s}\rangle$ by

$$
\left(\begin{array}{c}
|\eta\rangle \\
\left|\eta^{\prime}\right\rangle
\end{array}\right)=\left(\begin{array}{cc}
\cos \phi & -\sin \phi \\
\sin \phi & \cos \phi
\end{array}\right)\left(\begin{array}{l}
\left|\eta_{q}\right\rangle \\
\left|\eta_{s}\right\rangle
\end{array}\right) .
$$

The decay constants $f_{\eta^{(\prime)}}^{q}$ and $f_{\eta^{(\prime)}}^{s}$, as well as the other hadronic parameters related to $\eta$ and $\eta^{\prime}$ can then be expressed in terms of two decay constants $f_{q, s}$ and the mixing angle $\phi$ [51]. The values of these three parameters have been determined from a fit to experimental data, yielding [56]

$$
\begin{aligned}
f_{q} & =(1.07 \pm 0.02) f_{\pi}, \\
f_{s} & =(1.34 \pm 0.06) f_{\pi}, \\
\phi & =39.3^{\circ} \pm 1.0^{\circ} .
\end{aligned}
$$

Finally, a summary of the other input parameters entering our numerical analysis is given in Table 1 . It is noted that the latest experimental determinations of $f_{\pi}$ and $f_{K}$ [54] compare positively within errors with the lattice results [59]. Our values of the vector-meson decay constants are taken from [52], which are an update of the ones extracted in [53]. The scale dependence of the transverse decay constants is taken into account via the leading-logarithmic running $f_{\perp}(\mu)=f_{\perp}\left(\mu_{0}\right)\left[\alpha_{s}(\mu) / \alpha_{s}\left(\mu_{0}\right)\right]^{4 / 23}$. The light quark masses given in the table are the running masses defined in the $\overline{\mathrm{MS}}$ scheme; to get the corresponding pole and running masses at different scales, we use the NLO running formulae collected, for example, in [60]. The $b$ and $c$ quark masses are, however, defined as the pole masses. 


\section{Competing Interests}

The author declares that there is no conflict of interests regarding the publication of this paper.

\section{Acknowledgments}

This work is supported by CCNU-QLPL Innovation Fund (QLPL201411).

\section{References}

[1] C. H. Chang and Y. Q. Chen, "Decays of the $B_{c}$ meson," Physical Review D, vol. 49, no. 7, pp. 3399-3411, 1994.

[2] N. Brambilla, M. Krämer, R. Mussa et al., "Heavy quarkonium physics," https://arxiv.org/abs/hep-ph/0412158.

[3] F. Abe, H. Akimoto, A. Akopian et al., "Observation of the $B_{c}$ Meson in $p \bar{p}$ collisions at $\sqrt{s}=1.8 \mathrm{TeV}$," Physical Review Letters, vol. 81, no. 12, pp. 2432-2437, 1998.

[4] T. Aaltonen, J. Adelman, T. Akimoto et al., "Observation of the Decay $B_{c}^{ \pm} \rightarrow J / \psi \pi^{ \pm}$and Measurement of the $B_{c}^{ \pm}$Mass," Physical Review Letters, vol. 100, no. 18, Article ID 182002, 2008.

[5] V. M. Abazov, B. Abbott, M. Abolins et al., "Observation of the $B_{c}$ Meson in the Exclusive Decay $B_{c} \rightarrow J / \psi \pi$," Physical Review Letters, vol. 101, no. 1, Article ID 012001, 2001.

[6] R. Aaij, C. Abellan Beteta, A. Adametz et al., "Measurements of $B_{c}^{+}$production and mass with the $B_{c}^{+} \rightarrow J / \psi \pi^{+}$decay," Physical Review Letters, vol. 109, Article ID 232001, 2012.

[7] V. M. Abazov, K. A. Bloom, and G. Snow, "Measurement of the Lifetime of the $B_{c}^{ \pm}$Meson in the Semileptonic Decay Channel," Physical Review Letters, vol. 102, no. 9, Article ID 092001, 2009.

[8] A. Abulencia, D. Acosta, J. Adelman et al., "Measurement of the $B_{c}^{+}$Meson lifetime using the decay mode $B_{c}^{+} \rightarrow J \psi e^{+} v_{e}$," Physical Review Letters, vol. 97, no. 1, Article ID 012002, 7 pages, 2006.

[9] R. Aaij, B. Adeva, M. Adinolfi et al., "Measurement of the $B_{c}^{+}$ meson lifetime using $B_{c}^{+} \rightarrow J / \psi \pi^{+} \nu_{\mu} X$ decays," The European Physical Journal C, vol. 74, p. 2839, 2014.

[10] M. P. Altarelli and F. Teubert, "B physics AT LHCb," International Journal of Modern Physics A, vol. 23, no. 32, pp. 5117-5136, 2008.

[11] R. Aaij, C. Abellan Beteta, B. Adeva et al., "First observation of the decay $B_{c}^{+} \rightarrow J / \psi K^{+}$, Journal of High Energy Physics, vol. 2013, no. 9, article 075, 2013.

[12] R. Aaij, B. Adeva, M. Adinolfi et al., "Observation of the decay $B_{c}^{+} \rightarrow B_{s}^{0} \pi^{+}$, Physical Review Letters, vol. 111, no. 18, Article ID 181801, 2013.

[13] A. Bharucha, I. I. Bigi, C. Bobeth et al., "Implications of LHCb measurements and future prospects," The European Physical Journal C, vol. 73, article 2373, 2013.

[14] J. F. Sun, Y. L. Yang, W. J. Du, and H. L. Ma, "Study of $B_{c} \rightarrow$ $B^{(*)} P, B V$ decays with QCD factorization," Physical Review D, vol. 77, Article ID 114004, 2008.

[15] T. Aaltonen, S. Amerio, D. Amidei et al., "Evidence for the charmless annihilation decay mode $B_{s}^{0} \rightarrow \pi^{+} \pi^{-}$, Physical Review Letters, vol. 108, no. 21, Article ID 211803, 2012.

[16] F. Ruffini, "Measurements of charmless b-hadron decays at CDF; first evidence for the annihilation $B_{s}^{0} \rightarrow \pi^{+} \pi^{-}$decay mode," http://inspirehep.net/record/1233813.
[17] R. Aaij, C. Abellan Beteta, B. Adeva et al., "Measurement of bhadron branching fractions for two-body decays into charmless charged hadrons," Journal of High Energy Physics, vol. 2012, article 37, 2012.

[18] M. Bauer, B. Stech, and M. Wirbel, "Exclusive non-leptonic decays of $D^{-}, D_{S^{-}}$and $B$-mesons," Zeitschrift für Physik $C$, vol. 34, no. 1, pp. 103-115, 1987.

[19] Y.-Y. Keum, H.-N. Li, and A. I. Sanda, "Fat penguins and imaginary penguins in perturbative QCD," Physics Letters B, vol. 504, no. 1-2, pp. 6-14, 2001.

[20] M. Beneke, G. Buchalla, M. Neubert, and C. T. Sachrajda, "QCD factorization for exclusive non-leptonic B-meson decays: general arguments and the case of heavy-light final states," Nuclear Physics B, vol. 591, no. 1-2, pp. 313-418, 2000.

[21] M. Beneke, G. Buchalla, M. Neubert, and C. T. Sachrajda, "QCD factorization in $B \rightarrow \pi K, \pi \pi$ decays and extraction of Wolfenstein parameters," Nuclear Physics B, vol. 606, no. 1-2, pp. 245-321, 2001.

[22] C. W. Bauer, S. Fleming, D. Pirjol, and I. W. Stewart, "An effective field theory for collinear and soft gluons: heavy to light decays," Physical Review D, vol. 63, Article ID 114020, 2001.

[23] C. M. Arnesen, Z. Ligeti, I. Z. Rothstein, and I. W. Stewart, "Power corrections in charmless nonleptonic $B$ decays: annihilation is factorizable and real," Physical Review D, vol. 77, no. 5, Article ID 054006, 2008.

[24] D. Du, J. Sun, D. Yang, and G. Zhu, "Charmless two-body $B$ decays: a global analysis with QCD factorization," Physical Review D, vol. 67, no. 1, Article ID 014023, 11 pages, 2003.

[25] X. Liu, Z. J. Xiao, and C. D. Lu, "Pure annihilation type $B_{c} \rightarrow$ $M_{2} M_{3}$ decays in the perturbative QCD approach," Physical Review D, vol. 81, no. 1, Article ID 014022, 2010.

[26] S. Descotes-Genon, J. He, E. Kou, and P. Robbe, "Nonleptonic charmless $B_{c}$ decays and their search at LHCb," Physical Review $D$, vol. 80, Article ID 114031, 2009.

[27] Y. L. Yang, J. F. Sun, and N. Wang, "Study of $B_{c} \rightarrow K K$ decay with perturbative QCD approach," Physical Review D, vol. 81, no. 7, Article ID 074012, 2010.

[28] W. L. Ju, T. H. Wang, Y. Jiang, H. Yuan, and G. L. Wang, "The nonleptonic charmless decays of $B_{c}$ meson," http://arxiv.org/ abs/1512.05870.

[29] J. M. Cornwall, "Dynamical mass generation in continuum quantum chromodynamics," Physical Review D, vol. 26, no. 6, pp. 1453-1478, 1982.

[30] Q. Chang, X. Q. Li, and Y. D. Yang, "Revisiting $B \rightarrow \pi K, \pi K^{*}$ and $\rho K$ decays: CP violations and implication for new physics," JHEP: Journal of High Energy Physics, vol. 0809, p. 038, 2008.

[31] Q. Chang, X. W. Cui, L. Han, and Y. D. Yang, "Revisiting the annihilation corrections in nonleptonic $\vec{B}_{s}^{0}$ decays within QCD factorization," Physical Review D, vol. 86, no. 5, Article ID 054016, 2012.

[32] S. Bar-Shalom, G. Eilam, and Y. D. Yang, " $\vec{B} \varphi \pi$ and $B^{0} \rightarrow \varphi \varphi$ in the standard model and new bounds on $R$-parity violation," Physical Review D, vol. 67, Article ID 014007, 2003.

[33] Y. D. Yang, F. Su, G. R. Lu, and H. J. Hao, "Revisiting the annihilation decay $\bar{B}_{s} \rightarrow \pi^{+} \pi^{-}$," The European Physical Journal C, vol. 44, no. 2, pp. 243-247, 2005.

[34] Q. Chang and Y.-D. Yang, "The effects of a family non-universal $\mathrm{Z}^{\prime}$ boson in the $\bar{B}_{s} \rightarrow \pi K, \pi K^{*}, \rho K$ decays and $B_{d}-\bar{B}_{d}$ mixing," Nuclear Physics B, vol. 852, no. 3, pp. 539-552, 2011.

[35] A. J. Buras, "Weak Hamiltonian, CP violation and rare decays," https://arxiv.org/abs/hep-ph/9806471. 
[36] M. Kobayashi and T. Maskawa, " $C P$-violation in the renormalizable theory of weak interaction," Progress of Theoretical Physics, vol. 49, no. 2, pp. 652-657, 1973.

[37] K. Wang and G. Zhu, "Flavor dependence of annihilation parameters in QCD factorization," Physical Review D, vol. 88, no. 1, Article ID 014043, 2013.

[38] M. Beneke, X. Q. Li, and L. Vernazza, "Hadronic B decays in the MSSM with large $\tan \beta$," The European Physical Journal C, vol. 61, no. 3, pp. 429-438, 2009.

[39] S. J. Brodsky and C. Ji, "Exclusive Production of HigherGeneration Hadrons and Form-Factor Zeros in Quantum Chromodynamics," Physical Review Letters, vol. 55, no. 21, pp. 2257-2260, 1985 .

[40] J. Sun, Y. Yang, Q. Chang, and G. Lu, "Phenomenological study of the $B_{c} \rightarrow B P, B V$ decays with perturbative QCD approach," Physical Review D, vol. 89, Article ID 114019, 2014.

[41] D. Ebert, R. N. Faustov, and V. O. Galkin, "Spectroscopy and Regge trajectories of heavy quarkonia and $B_{c}$ mesons," The European Physical Journal C, vol. 71, article 1825, 2011.

[42] G. Eilam, M. Ladisa, and Y. D. Yang, "Study of $B^{0} \rightarrow J / \psi D^{(*)}$ and $\eta_{c} D^{(*)}$ in perturbative QCD," Physical Review D, vol. 65, Article ID 037504, 2002.

[43] P. Ball, V. M. Braun, Y. Koike, and K. Tanaka, "Higher twist distribution amplitudes of vector mesons in QCD: formalism and twist 3 distributions," Nuclear Physics B, vol. 529, no. 1-2, pp. 323-382, 1998.

[44] A. C. Aguilar, A. A. Natale, and P. S. Rodrigues da Silva, "Relating a gluon mass scale to an infrared fixed point in pure gauge QCD," Physical Review Letters, vol. 90, no. 15, Article ID 152001, 2003.

[45] R. Aouane, F. Burger, E. Ilgenfritz, M. Müller-Preussker, and A. Sternbeck, "Landau gauge gluon and ghost propagators from lattice QCD with $N_{f}=2$ twisted mass fermions at finite temperature," Physical Review D, vol. 87, no. 11, Article ID 114502, 2013.

[46] S. Gongyo and H. Suganuma, "Gluon propagators in maximally Abelian gauge in SU(3) lattice QCD," Physical Review D, vol. 87, Article ID 074506, 2013.

[47] J. M. Cornwall, "Positivity violations in qcd," Modern Physics Letters A, vol. 28, no. 38, Article ID 1330035, 17 pages, 2013.

[48] R. Alkofer and L. von Smekal, “The infrared behaviour of QCD Green's functions: confinement, dynamical symmetry breaking, and hadrons as relativistic bound states," Physics Reports A: Review Section of Physics Letters, vol. 353, no. 5-6, pp. 281-465, 2001.

[49] C. S. Fischer, A. Maas, and J. M. Pawlowski, "On the infrared behavior of Landau gauge Yang-Mills theory," Annals of Physics, vol. 324, no. 11, pp. 2408-2437, 2009.

[50] P. Boucaud, J. P. Leroy, A. Le Yaouanc, J. Micheli, O. Pène, and J. Rodríguez-Quintero, "The infrared behaviour of the pure yangmills green functions," Few-Body Systems, vol. 53, no. 3-4, pp. 387-436, 2012.

[51] M. Beneke and M. Neubert, "Flavor-singlet B-decay amplitudes in QCD factorization," Nuclear Physics B, vol. 651, no. 1-2, pp. 225-248, 2003.

[52] M. Jung, X.-Q. Li, and A. Pich, "Exclusive radiative B-meson decays within the aligned two-Higgs-doublet model," Journal of High Energy Physics, vol. 2012, article 63, 2012.

[53] P. Ball, G. W. Jones, and R. Zwicky, " $B \rightarrow V_{\gamma}$ beyond QCD factorization," Physical Review D, vol. 75, no. 5, Article ID 054004, 2007.
[54] K. A. Olive, S. Golwala, P. Vogel et al., "Review of particle physics," Chinese Physics C, vol. 38, no. 9, Article ID 090001, 2014.

[55] T. W. Chiu, T.-H. Hsiehb, C.-H. Huanga, and K. Ogawa, "Beauty mesons in lattice QCD with exact chiral symmetry," Physics Letters B, vol. 651, no. 2-3, pp. 171-176, 2007.

[56] T. Feldmann, P. Kroll, and B. Stech, "Mixing and decay constants of pseudoscalar mesons," Physical Review D, vol. 58, no. 11, Article ID 114006, 1998.

[57] L. Wolfenstein, "Parametrization of the Kobayashi-Maskawa Matrix," Physical Review Letters, vol. 51, no. 21, pp. 1945-1947, 1983.

[58] J. Charles, A. Höcker, H. Lacker et al., " $C P$ violation and the CKM matrix: assessing the impact of the asymmetric B factories," The European Physical Journal C: Particles and Fields, vol. 41, no. 1, pp. 1-131, 2005.

[59] S. Aoki, Y. Aoki, C. Bernard et al., "Review of lattice results concerning low-energy particle physics," The European Physical Journal C, vol. 74, article 2890, 2014.

[60] K. G. Chetyrkin, J. H. Kuhn, and M. Steinhauser, "RunDec: a mathematica package for running and decoupling of the strong coupling and quark masses," Computer Physics Communications, vol. 133, no. 1, pp. 43-65, 2000. 

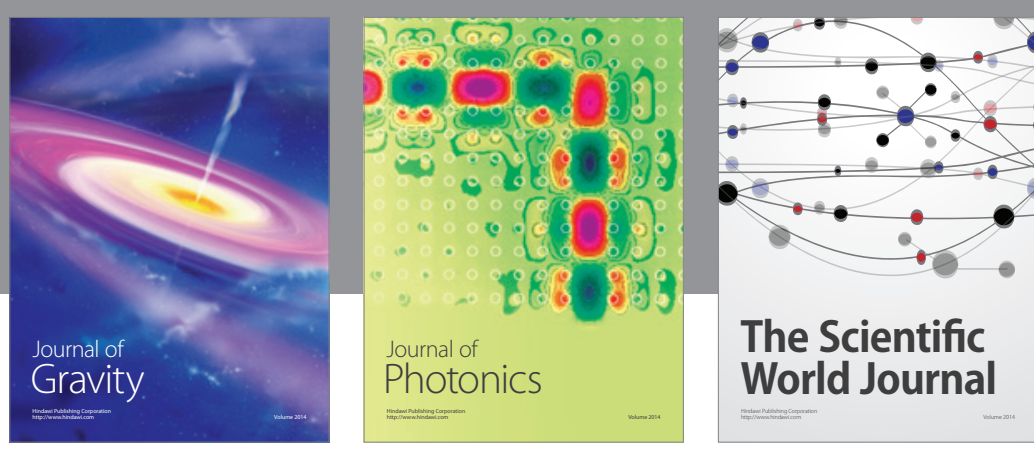

The Scientific World Journal
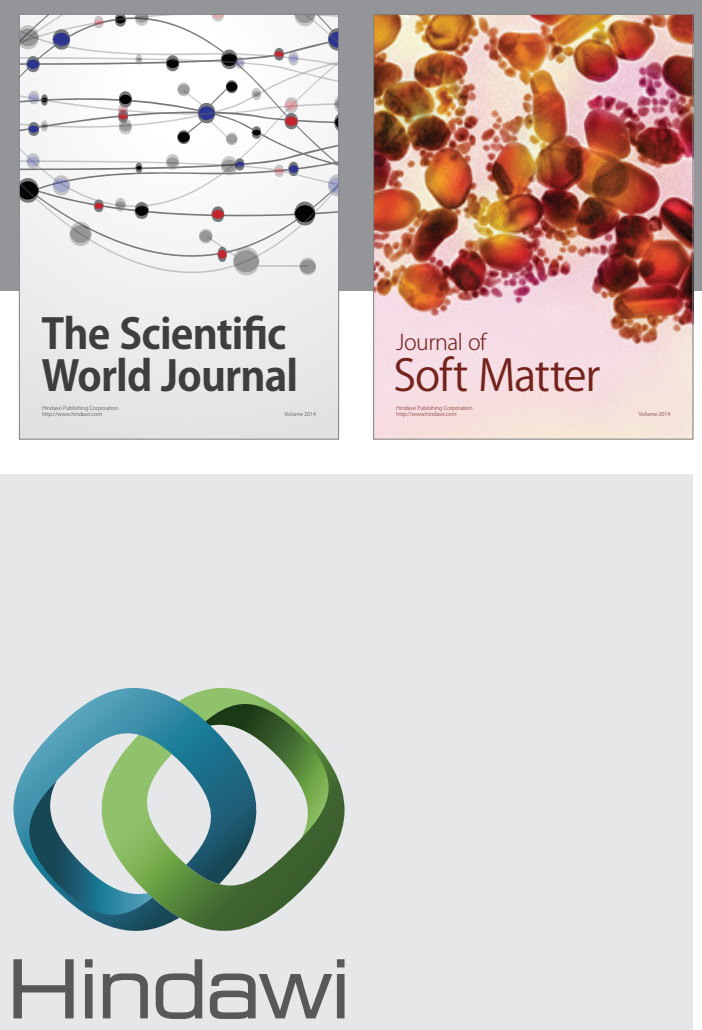

Submit your manuscripts at

http://www.hindawi.com

nternational Journal of

Statistical Mechanics
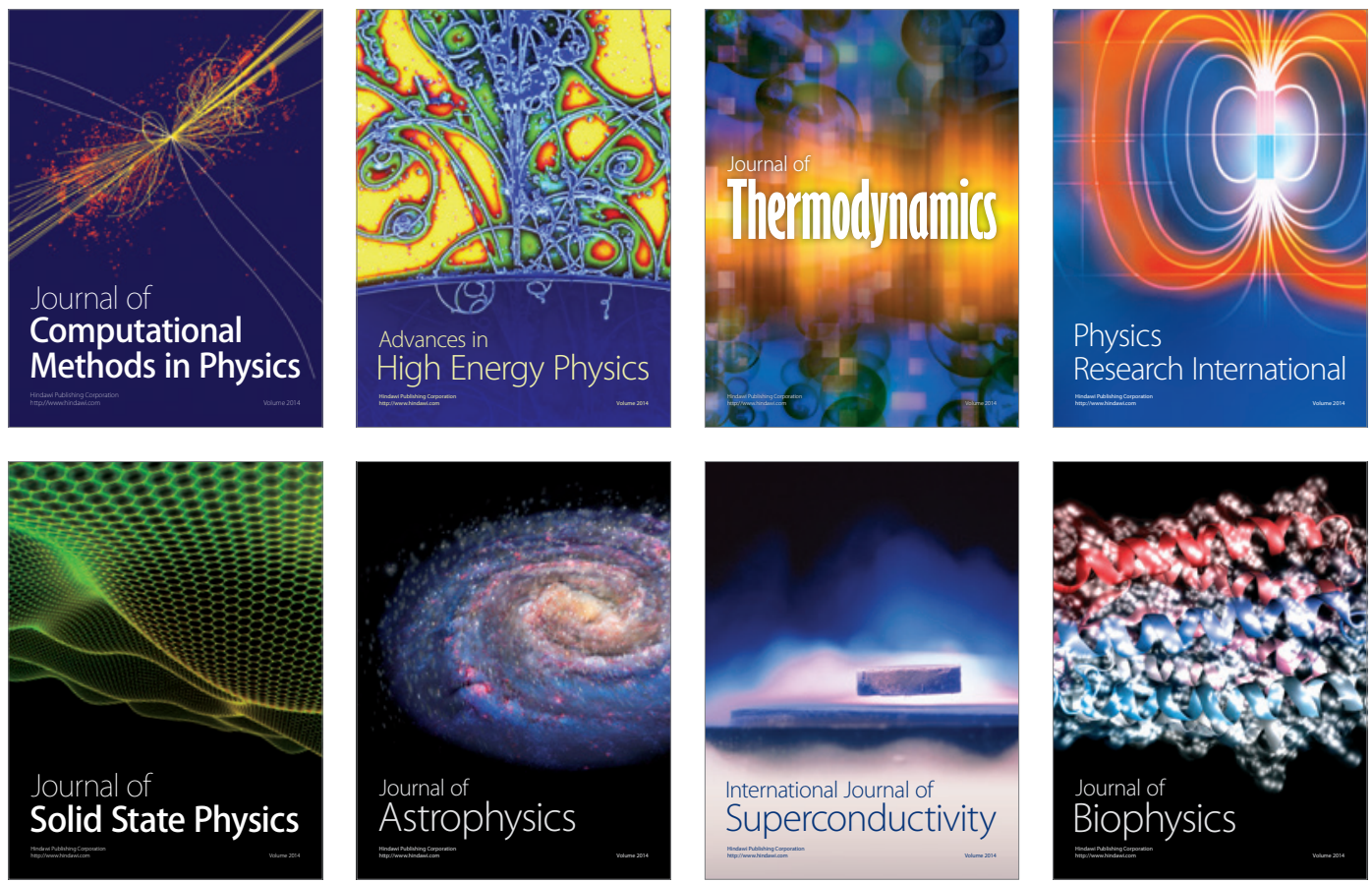
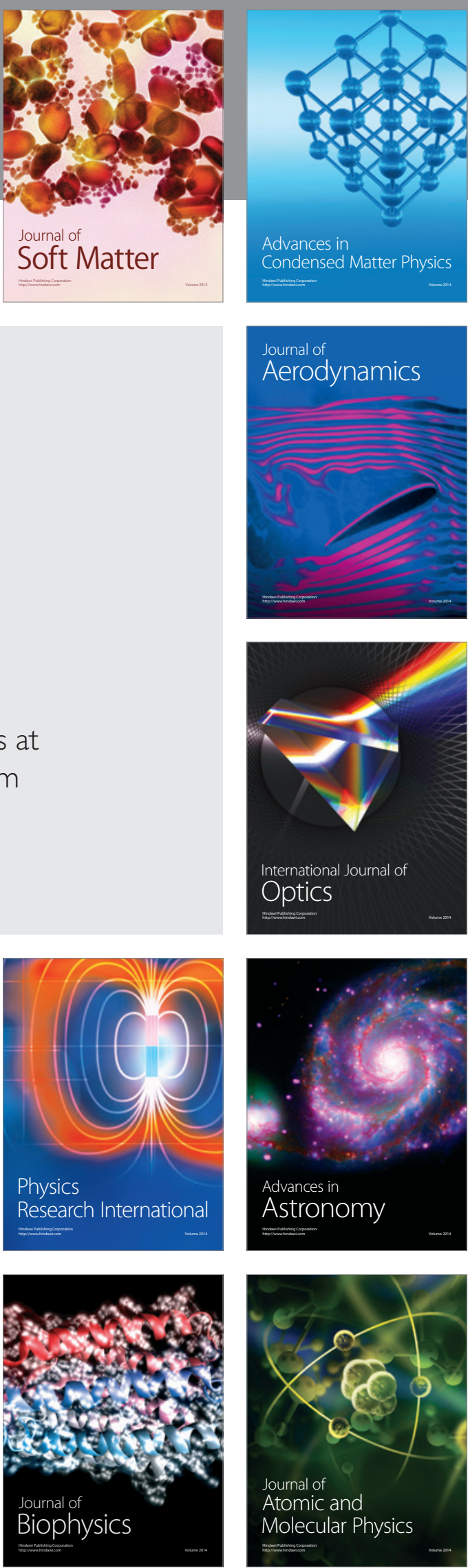